\title{
REPRESENTATION OF A 2-POWER AS SUM OF $k$ 2-POWERS: THE ASYMPTOTIC BEHAVIOR
}

\author{
G. MOLTENI
}

\begin{abstract}
A $k$-representation of an integer $\ell$ is a representation of $\ell$ as sum of $k$ powers of 2 , where representations differing by the order are considered as distinct. Let $\mathcal{W}(\sigma, k)$ be the maximum number of such representations for integers $\ell$ whose binary representation has exactly $\sigma$ non-zero digits. $\mathcal{W}(\sigma, k)$ can be recovered from $\mathcal{W}(1, k)$ via an explicit formula, thus in some sense $\mathcal{W}(1, k)$ is the fundamental object. In this paper we prove that $(\mathcal{W}(1, k) / k !)^{1 / k}$ tends to a computable limit as $k$ diverges. This result improves previous bounds which were obtained with purely combinatorial tools.
\end{abstract}

Int. J. Number Theory 8(8), 1923-1963 (2012).

\section{INTRODUCTION AND MAIN RESULT}

Given an integer $m \geq 2$, the $m$-ary Euler partition problem is a well-known combinatorial problem dealing with the study of the number of representations of an integer $\ell$ as sum of integral, nonnegative and non-decreasing powers of $m$, i.e. with the quantities:

$$
b_{m, \infty}(\ell):=\sharp\left\{\text { sequences } a_{n} \in \mathbb{N} \cup\{0\} \forall n: \sum_{n=0}^{\infty} a_{n} m^{n}=\ell\right\} .
$$

The asymptotic behavior of $b_{m, \infty}(\ell)$ as $\ell$ diverges has been described independently by many authors, such as Mahler [11], de Bruijn [2] and Knuth [9], who have found it using different approaches that, however, are all rooted in the special form of its generating function. The same result was also discussed in a paper by Erdős [3], who proved it with elementary tools, and it can be deduced easily by the general tauberian theorem of Ingham [8].

Reznick [16] has considered an interesting modification of the problem for the case of $m=2$, introducing a further integral parameter $d$ and imposing the restriction $a_{n}<d$ to the coefficients in (1); the new sequences are then denoted by $b_{2, d}(\ell)$. The parameter $d$ adds a new level of complexity to the problem since the asymptotic behavior of $b_{2, d}(\ell)$ changes considerably according to the parity of $d$ : in fact, he proved that the limit of the quotient $\log \left(b_{2, d}(\ell)\right) / \log \ell$ exists when $d$ is even, but that, for $d=3$, it oscillates in a bounded range $\left[\lambda_{1}, \lambda_{2}\right]$ with $\lambda_{1}<\lambda_{2}$, and suggested that this phenomenon should persist for every odd $d \geq 3$ with constants depending on $d$. This conjecture was recently proved by Protasov [14, 15], who also described an algebraic characterization of the $\lambda_{j}$ constants allowing their systematic computation in principle, and their effective computation for several values of $d$.

Another variation of this sort of problems is the following. We call $k$-representation of an integer $\ell$ any string $\boldsymbol{n}=\left(n_{1}, \ldots, n_{k}\right)$ of non-negative integers such that

2010 Mathematics Subject Classification. Primary 11 B 75, Secondary 11 A 99.

Key words and phrases. $k$-representations, binary partition problem. 
$\sum_{j=1}^{k} 2^{n_{j}}=\ell$, and $\mathcal{U}(\ell, k)$ is the number of $k$-representations of $\ell$ so that:

$$
\mathcal{U}(\ell, k):=\sharp\left\{\boldsymbol{n}=\left(n_{1}, \ldots, n_{k}\right), n_{j} \in \mathbb{N} \cup\{0\} \forall j: \sum_{j=1}^{k} 2^{n_{j}}=\ell\right\} .
$$

The study of $\mathcal{U}(\ell, k)$ for a given $k$ as a function of $\ell$ is a problem that has some similarities with the constants $b_{2, d}(\ell)$. In a recent paper [12], we met the necessity for the study of the opposite situation where both $\ell$ and $k$ diverge. In fact, in that work, an upper bound for $\max _{\ell}\{\mathcal{U}(\ell, k)\}$ as a function of $k$ was the main tool for the proof of a nontrivial cancellation in certain short exponential sums. Recently, we discovered that the same quantities already appeared in a joint paper of Lehr, Shallit and Tromp [10], who proved the upper bound $\max _{\ell}\{\mathcal{U}(\ell, k)\} \ll k ! 2^{k}$. In [12], we proved a better bound using a different approach: we discuss it briefly now since it is necessary for the comprehension of the content of the present paper.

For every fixed $k$, the chaotic behavior of $\mathcal{U}(\ell, k)$ with respect to $\ell$ disappears when $\ell$ is selected within a set of integers all having the same number of ones in their binary representation. This fact suggests defining the quantity:

$$
\mathcal{W}(\sigma, k):=\max _{\ell: \sigma(\ell)=\sigma}\{\mathcal{U}(\ell, k)\},
$$

where $\sigma(\ell)$ counts the number of ones appearing in the binary representation of $\ell$. There is a simple formula connecting $\mathcal{W}(\sigma, k)$ to $\mathcal{W}(1, k)$ (see [12] for a proof):

$$
\frac{\mathcal{W}(\sigma, k)}{k !}=\sum_{\substack{k_{1}, \ldots, k_{\sigma} \geq 1 \\ k_{1}+\cdots+k_{\sigma}=k}} \prod_{j=1}^{\sigma} \frac{\mathcal{W}\left(1, k_{j}\right)}{k_{j} !},
$$

that can also be written in the following iterative way:

$$
\frac{\mathcal{W}(\sigma, k)}{k !}=\sum_{n=1}^{k-1} \frac{\mathcal{W}(1, n)}{n !} \cdot \frac{\mathcal{W}(\sigma-1, k-n)}{(k-n) !} .
$$

The definition of $\mathcal{W}(1, k)$ as $\max _{w}\left\{\mathcal{U}\left(2^{w}, k\right)\right\}$ is not suitable for the computation of its value; however, it has been proven that the maximum is reached for every $w \geq k-1$ (see [12], but the same result is also given in [10]), and, based upon this fact, in a joint paper with A. Giorgilli [5] we proved the following recursive formula:

Theorem. Let $M_{k, l}$ be the two indexes sequence defined as

$$
\begin{array}{ll}
M_{k, l}=0 & \text { if } l \geq k, \\
M_{k, k-1}=1 & \text { if } k>1, \\
M_{k, l}=\sum_{s=1}^{2 l}\left(\begin{array}{c}
k+l-1 \\
2 l-s
\end{array}\right) M_{k-l, s} & \text { if } 1 \leq l<k-1 .
\end{array}
$$

Then $\mathcal{W}(1, k)=M_{k, 1}$ for all $k>1$.

The previous formulas allow us to compute very efficiently the values of $\max _{\ell}\{\mathcal{U}(\ell, k)\}$ also for large $k$. This was an important fact that we used widely in [12] for a computational part needed for the proof of the bounds:

$$
(1.75218)^{k} \ll \max _{\ell}\{\mathcal{U}(\ell, k)\} / k ! \leq(1.75772)^{k} .
$$


A second ingredient for the proof of (4) was the explicit bounds:

$$
0.3316 \cdot(1.1305)^{k} \leq \mathcal{W}(1, k) / k ! \leq(1.71186)^{k-1} \cdot k^{-1.6}
$$

that we proved with combinatorial tools (Theorem 13 in [10] gives the weaker bound $\left.\mathcal{W}(1, k) / k ! \ll 1.8^{k}\right)$. Inequalities (4) and (5) were suitable for the applications in [12] but are not conclusive regarding the behavior of $\mathcal{W}(1, k)$ and $\mathcal{U}(\ell, k)$ : numerical tests show that $(\mathcal{W}(1, k) / k !)^{1 / k}$ tends very quickly toward a constant whose approximated value is 1.19, while the bounds in (5) are far away from this value. According to [10], this fact was mentioned by Knuth in a private communication with R. E. Tarjan, where he also suggested the asymptotic behavior $\mathcal{W}(1, k) \sim c_{1} k^{k-c_{2}}$ for a suitable couple of positive constants $c_{1}$ and $c_{2}$. The aim of the present paper is to prove the following result.

Theorem 1. The limit of $(\mathcal{W}(1, k) / k !)^{1 / k}$ when $k$ diverges exists, it can be computed with arbitrary precision, and

$$
1.192674341213466032221288982528755 \ldots
$$

is its value with thirty four correct digits.

Note that this result disproves the Knuth's conjecture.

From (2), we obtain almost immediately the following generalization.

Corollary 1. The limit of $(\mathcal{W}(\sigma, k) / k !)^{1 / k}$ when $k$ diverges exists for every fixed $\sigma$ and is independent of $\sigma$ (and therefore coincides with the constant introduced in the previous theorem).

In spite of this success in the improvement of (5), the theorem is not sufficient to also improve (4). In fact, the value of $\sigma$ giving the maximum in (2) is essentially of the same order of $k$ so that $\left(\max _{\ell}\{\mathcal{U}(\ell, k)\}\right)^{1 / k}=\left(\max _{\sigma}\{\mathcal{W}(\sigma, k)\}\right)^{1 / k}$ is affected not only by $\lambda:=\lim _{k}(\mathcal{W}(1, k) / k !)^{1 / k}$ but also by the upper/lower limits of $\mathcal{W}(1, k) / \lambda^{k} k !$; in fact, numerical tests suggest that this quotient tends to a number whose value is approximatively 0.248 , but unfortunately, our argument does not give any indication about the existence of these constants.

The very simple formulation of the theorem is not reflected into its proof, that is actually quite intricate. Roughly, it runs as follows: we introduce a family of matrices $S_{\ell}$, and the numbers $\Lambda_{r, \ell}:=\left\|S_{\ell}^{r}\right\|^{1 / r}, \lambda_{\infty}:=\lim _{\ell \rightarrow \infty} \lim _{r \rightarrow \infty} \Lambda_{r, \ell}$, and $\Lambda_{\infty}:=\lim _{r \rightarrow \infty} \lim _{\ell \rightarrow \infty} \Lambda_{r, \ell}$. We notice that $\lambda_{\ell}:=\lim _{r \rightarrow \infty} \Lambda_{r, \ell}$ is the spectral radius of $S_{\ell}$, so that $\lambda_{\infty}$ is the limit of the spectral radii for the matrices $S_{\ell}$; also $\Lambda_{\infty}$ admits a similar interpretation for a certain element in a suitable infinite dimensional Banach algebra. Then we prove that $\lambda_{\infty}$ is a lower bound for $\lim _{\inf }{ }_{k \rightarrow \infty}(\mathcal{W}(1, k) / k !)^{1 / k}$ and that $\Lambda_{\infty}$ is an upper bound for $\lim \sup _{k \rightarrow \infty}(\mathcal{W}(1, k) / k !)^{1 / k}$; thus, $\lambda_{\infty}$ is not greater than $\Lambda_{\infty}$ and the theorem follows by the proof of their equality. For this task, we introduce a second family of matrices $\mathcal{F}_{\ell}(x)$ depending on a real parameter $x$ and whose spectral radius is 1 for a unique value $x_{\ell}$. The matrices $\mathcal{F}_{\ell}\left(x_{\ell}\right)$ are very similar to the transpose of the original matrices $S_{\ell}$, and this implies that the constants $x_{\ell}$ are asymptotically equal to $1 / \sqrt{\lambda_{\ell}}$ and, hence, tend to $1 / \sqrt{\lambda_{\infty}}$. Finally, and this is the most complicated part of the argument, we show that $\Lambda_{\infty}$ is estimated from above by $\left(x_{\ell}+o(1)\right)^{-2}$ for infinitely many $\ell$ so that $\Lambda_{\infty} \leq \lambda_{\infty}$. This allows us to conclude the proof since we have already proved the opposite inequality.

The proof is given in four distinct sections: the general setting in Section 2, the lower bound in Sections 3 (where the existence of $\lambda_{\infty}$ and the validity of the lower 
inequality are proved) and 4 (where $\lambda_{\infty}$ is computed), and the upper bound and the equality of $\lambda_{\infty}$ and $\Lambda_{\infty}$ in Section 5 .

It is a tantalizing coincidence that the recent results of Protasov $[14,15]$ are also based upon the spectral properties of a family of nonnegative matrices.

Many results that we prove in the following sections are given in a form that is strictly related to the task of present paper, but we strongly believe that they could be generalized to other problems: we leave such a general setting to a future paper.

\section{Preliminary facts}

We need to address some terminology and definitions. The symbol $\left[\frac{1}{n}\right]_{\ell}$ denotes the horizontal vector $\left(\frac{1}{n !}, \frac{1}{(n-1) !}, \ldots\right)$ with $\ell$ entries, where $\frac{1}{m !}$ is set to zero when $m<0 ; \mathbb{I}_{n}$ is the identity matrix of order $n$ and $O_{m \times n}$ is the $m \times n$ null matrix; $(n)_{m}$ is the descending Pochhammer symbol, i.e., $(n)_{0}:=1$ and $(n)_{m}=n(n-1)_{m-1}$ when $m \geq 1 ;\lfloor x\rfloor$ denotes the maximum of $\{n \in \mathbb{Z}: n \leq x\}$ and $\lceil x\rceil$ the minimum of $\{n \in \mathbb{Z}: n \geq x\}$; given two square matrices $A$ and $B$ of order $l_{A}$ and $l_{B}$, respectively, and having nonnegative entries, the notation $A \leq B$ means that $l_{A} \leq l_{B}$ and that $B$ contains a principal submatrix $B^{\prime}$ of order $l_{A}$ such that the entries of $B^{\prime}-A$ are nonnegative; given a vector $\boldsymbol{u} \in \mathbb{C}^{n}$ and a matrix $A,\|\boldsymbol{u}\|$ denotes the $\ell^{1}$-norm of $\boldsymbol{u}$ and $\|A\|_{k}$ the $\ell^{1}$-norm of the $k$ th column of $A$; the norm of $A$ is defined as $\|A\|:=\sup _{\|\boldsymbol{u}\|=1}\|A \boldsymbol{u}\|$ and can be computed as the maximum of the norms of its columns. Moreover, for every integer $\ell \geq 2$ and for every $u=1, \ldots, \ell$, we define $A_{u}^{(\ell)}$ as the square matrix of order $\ell$ whose $u$ th row is $\left[\frac{1}{2 u-1}\right]_{\ell}$ and every other row is zero. Finally, $S_{\ell}$ denotes the square matrix of order $\ell^{2}$ :

$$
S_{\ell}:=\left(\begin{array}{cccc}
A_{1}^{(\ell)} & A_{2}^{(\ell)} & \cdots & A_{\ell}^{(\ell)} \\
& \mathbb{I}_{(\ell-1) \ell} & & O_{(\ell-1) \ell \times \ell}
\end{array}\right),
$$

so that, for example

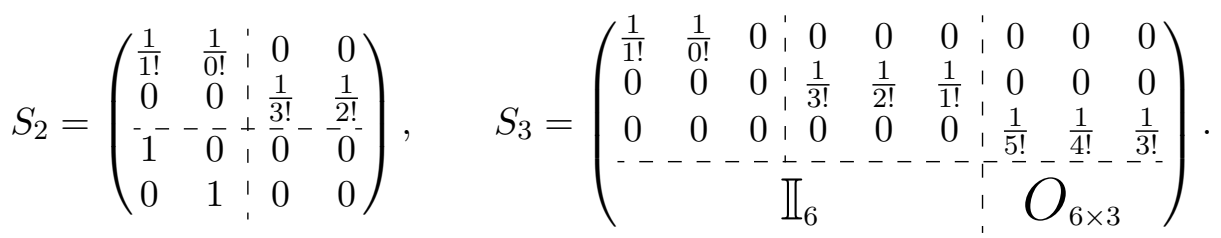

The fact that representations differing by order are considered distinct in $\mathcal{W}(1, k)$ implies that it grows at least as $k$ !. It is convenient to eliminate this factorial term at the beginning, turning our attention to the quotient $\mathcal{W}(1, k) / k$ !; for this reason, we introduce the new sequence $N_{k, l}:=M_{k, l} /(k+l-1)$ ! so that $\mathcal{W}(1, k) / k !=N_{k, 1}$. The formulas in (3), when written for $N_{k, l}$, become:

$$
\begin{array}{ll}
N_{k, l}=0 & \text { if } l \geq k, \\
N_{k, k-1}=1 /(2 k-2) ! & \text { if } k>1, \\
N_{k, l}=\sum_{s=1}^{2 l} \frac{N_{k-l, s}}{(2 l-s) !} & \text { if } 1 \leq l<k-1 .
\end{array}
$$

These recursive relations involve infinitely many variables. We simplify them by introducing a "cut-off" parameter, but we need a preliminary result giving a weak upper bound for $N_{k, l}$. 
Lemma 1. $N_{k, l} \leq \frac{2^{k-3}}{l !^{2}}$ for every $k$ and every $l$.

Better bounds can be proved with a more complicated argument, but they do not simplify the proof of the theorem in any stage.

Proof. The proof is by induction on $k$. For $k=1$, and for $k \geq 2, l \geq k$ the claim is evident because $N_{k, l}=0$. It is true also for $k=2$ and $l=1$, as equality. Let $\bar{k} \geq 3$ and suppose the claim for every $k<\bar{k}$. The claim for $l=\bar{k}-1$ states (by (6b)) that $\frac{1}{(2 k-2) !} \leq \frac{2^{\bar{k}-3}}{((k-1) !)^{2}}$, i.e. that $2^{\bar{k}-3}\left(\begin{array}{c}2 \bar{k}-2 \\ \bar{k}-1\end{array}\right) \geq 1$, which is true. Finally, for $l<\bar{k}-1$ and by induction and $(6 \mathrm{c})$ we have:

$$
N_{\bar{k}, l}=\sum_{s=1}^{2 l} \frac{N_{\bar{k}-l, s}}{(2 l-s) !} \leq \sum_{s=1}^{2 l} \frac{2^{\bar{k}-l-3}}{(2 l-s) ! s !^{2}}<\frac{2^{\bar{k}-l-3}}{(2 l) !} \sum_{s=0}^{2 l}\left(\begin{array}{c}
2 l \\
s
\end{array}\right)=\frac{2^{\bar{k}+l-3}}{(2 l) !} \leq \frac{2^{\bar{k}-3}}{l !^{2}} .
$$

For every fixed integer $\ell>1$, the "cut-off" parameter, let $a_{k, l}$ and $b_{k, l}$ be the sequences with $k \in \mathbb{N}$ and $l \leq \ell$ which are defined respectively by:

$$
\begin{array}{ll}
a_{k, l}=N_{k, l} & \text { if } k, l \leq \ell, \\
a_{k, l}=\sum_{s=1}^{\ell} \frac{a_{k-l, s}}{(2 l-s) !} & \text { if } l \leq \ell<k,
\end{array}
$$

and by:

$$
\begin{array}{rlrl}
b_{k, l} & =N_{k, l} & & \text { if } k, l \leq \ell, \\
b_{k, l}=\sum_{s=1}^{\ell} \frac{b_{k-l, s}}{(2 l-s) !}+\frac{2^{k}}{(\ell+1) !^{2}} \delta_{2 l>\ell} & \text { if } l \leq \ell<k,
\end{array}
$$

where in both $(7 \mathrm{~b})$ and $(8 \mathrm{~b})$ we have adopted our convention saying that $\frac{1}{(2 l-s) !}:=0$ when $2 l<s$. The numbers $a_{k, l}$ and $b_{k, l}$ are, respectively, a lower bound and an upper bound for $N_{k, l}$ : the first claim follows at once from the inequality $N_{k, l} \geq 0$, while the second one follows from Lemma 1, proving that the contribution of the $\operatorname{sum} \sum_{s=\ell+1}^{2 l} \frac{N_{k-l, s}}{(2 l-s) !}$ to the sum in (6c) is, at most:

$$
\sum_{s=\ell+1}^{2 l} \frac{N_{k-l, s}}{(2 l-s) !} \leq \sum_{s=\ell+1}^{2 l} \frac{2^{k-l-3}}{(2 l-s) ! s !^{2}} \leq \frac{2^{k-l-3}}{(\ell+1) !^{2}} \sum_{s=\ell+1}^{2 l} \frac{1}{(2 l-s) !} \leq \frac{2^{k}}{(\ell+1) !^{2}} .
$$

The recursive laws (7) and (8) can be written in a more explicit way. Let us extend the range of the sequences $N_{k, l}, a_{k, l}$ and $b_{k, l}$ to $k=0$ by setting $N_{0, l}:=a_{0, l}:=b_{0, l}:=$ 0 for every $l \leq \ell$, and let $\boldsymbol{a}_{k}$ and $\boldsymbol{b}_{k}$ in $\mathbb{C}^{\ell^{2}}$ be defined for $k \geq \ell$ as:

$$
\begin{aligned}
& \boldsymbol{a}_{k}:=\left(a_{k, 1}, \ldots, a_{k, \ell}, a_{k-1,1}, \ldots, a_{k-1, \ell}, a_{k-2,1}, \ldots, a_{k-\ell, \ell}\right)^{\top}, \\
& \boldsymbol{b}_{k}:=\left(b_{k, 1}, \ldots, b_{k, \ell}, b_{k-1,1}, \ldots, b_{k-1, \ell}, b_{k-2,1}, \ldots, b_{k-\ell, \ell}\right)^{\top} .
\end{aligned}
$$

Then (7) becomes:

$$
\begin{aligned}
& \boldsymbol{a}_{\ell}:=\left(N_{\ell, 1}, \ldots, N_{\ell, \ell}, N_{\ell-1,1}, \ldots, N_{\ell-1, \ell}, N_{\ell-2,1}, \ldots, N_{0, \ell}\right)^{\top}, \\
& \boldsymbol{a}_{k}=S_{\ell} \boldsymbol{a}_{k-1} \quad \text { if } k>\ell,
\end{aligned}
$$

and (8) becomes:

$$
\boldsymbol{b}_{\ell}:=\left(N_{\ell, 1}, \ldots, N_{\ell, \ell}, N_{\ell-1,1}, \ldots, N_{\ell-1, \ell}, N_{\ell-2,1}, \ldots, N_{0, \ell}\right)^{\top},
$$




$$
\boldsymbol{b}_{k}=S_{\ell} \boldsymbol{b}_{k-1}+\frac{2^{k}}{(\ell+1) !^{2}} \boldsymbol{z}, \quad \text { if } k>\ell,
$$

where

$$
\boldsymbol{z}:=(\underbrace{0, \ldots, 0}_{\lfloor\ell / 2\rfloor}, \underbrace{1, \ldots, 1}_{\lceil\ell / 2\rceil}, \underbrace{0, \ldots, 0}_{\ell^{2}-\ell})^{\top} .
$$

As we see, both (9) and (10) are linear recursive relations ruled by the same matrix $S_{\ell}$. Let $\lambda_{\ell}$ be the spectral radius of $S_{\ell}$; it controls the growth of the generic sequence $\boldsymbol{a}_{k}^{\prime}$ satisfying $\boldsymbol{a}_{k}^{\prime}=S_{\ell} \boldsymbol{a}_{k-1}^{\prime}$, but the sequence $\boldsymbol{a}_{k}$ comes with a specific initial condition (9a), and it is not evident that the spectral radius controls the growth of this particular sequence. We will prove that this follows by the non-negativity of $\boldsymbol{a}_{\ell}$ and the special structure of $S_{\ell}$. Moreover, the inhomogeneous part in (10b) grows as $2^{k} /(\ell+1) !^{2}$, while, in Section 4 , we will see that $\lambda_{\ell}$ is strictly lower than 1.2 . It follows that, for every fixed $\ell$, the growth of the solutions of (10b) is dominated by the exponential $2^{k}$, so no useful upper bound for $N_{k, l}$ can be obtained in this way. We will overcome this difficulty by taking advantage of the fact that the inhomogeneous part contains the term $(\ell+1) !^{2}$ in its denominator, so it can be small in size if we allow $\ell$ to grow with $k$. In other words, we will be able to prove the upper bound by exploring the uniformity of the solutions of (10) in $\ell$.

\section{LOWER BOUND}

The matrix $S_{\ell}$ is non-negative and irreducible; i.e., there is not a permutation $P$ such that $P S_{\ell} P^{\top}$ is block-triangular. In fact, this is equivalent to the following claim (see [19], Th. 1.6).

Proposition 1. The directed graph $G\left(S_{\ell}\right)$ associated with $S_{\ell}$ is strongly connected for every $\ell$.

The following diagram illustrates the claim for the matrices $S_{2}$ and $S_{3}$ :

$G\left(S_{2}\right)$ :
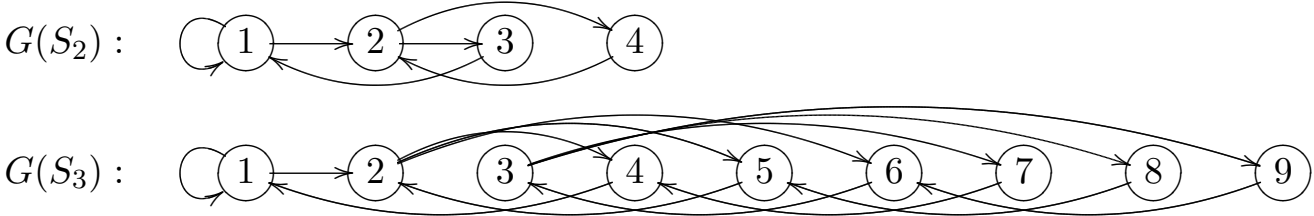

Proof. We divide the proof in three steps:

Step 1. It is sufficient to prove that every node $j$ with $j \leq \ell$ is connected to every node by a path, because every node $j$ with $j>\ell$ is directly connected by an arc to $j-\ell$.

Step 2. It is sufficient to prove that 1 is connected to every other node by a path, because the paths $2 \rightarrow \ell+1 \rightarrow 1,3 \rightarrow 2 \ell+1 \rightarrow \ell+1 \rightarrow 1,4 \rightarrow 3 \ell+1 \rightarrow$ $2 \ell+1 \rightarrow \ell+1 \rightarrow 1$, and so on, connect the nodes $2, \ldots, \ell$ to 1 .

Step 3. For every $r \leq \ell$, there is an arc from $r$ to each node in $\{(r-1) \ell+1, \ldots,(r-$ $1) \ell+\min (2 r, \ell)\}$. In particular, for every $r \leq \ell-1$, there is an arc from $r$ to $(r-1) \ell+r+1$ and, hence, a path from $r$ to $r+1$ (by Step 1). Linking together these paths, we get a new path from 1 to $\ell$, thus proving that there are paths from 1 to each node in $\{(\ell-1) \ell+1, \ldots,(\ell-1) \ell+\ell\}$. Let $q$ be any node and let $\bar{q} \in\{1, \ldots, \ell\}$ with $q=\bar{q}(\bmod \ell)$; we have just proved that there is a path from 1 to $\ell(\ell-1)+\bar{q}$ and, according to Step 1 , there is a 
path from $\ell(\ell-1)+\bar{q}$ to $q$; hence, the existence of a path from 1 to $q$ is also proved.

The irreducibility of $S_{\ell}$ and the fact that it has a non-zero element in its main diagonal (the upper left entry in $S_{\ell}$ is always 1) imply that $S_{\ell}$ is primitive, i.e., that $S_{\ell}^{r}$ is a positive matrix for some power $r$ (see [19], Th. 2.3). According to the Perron-Frobenius theorem (see [19], Th. 2.1), the irreducibility of $S_{\ell}$ implies that $\lambda_{\ell}$ is a simple eigenvalue of $S_{\ell}$, and its primitivity implies that every other eigenvalue has a strictly smaller absolute value. Furthermore, the definition of $S_{\ell}$ shows that $S_{\ell-1}$ is a principal submatrix of $S_{\ell}$ for every $\ell \geq 3$, i.e., that we recover $S_{\ell-1}$ from $S_{\ell}$ by erasing the $j$ th row and $j$ th column in $S_{\ell}$ for some set of $j$ s (for example, we recover $S_{2}$ erasing the $j$ th row and the $j$ th column in $S_{3}$ for $j \in\{3,6,7,8,9\}$ ). Under this condition, the Perron-Frobenius theorem also ensures that the spectral radius of $S_{\ell-1}$ is strictly lower than that of $S_{\ell}$; in other words, the sequence $\left\{\lambda_{\ell}\right\}_{\ell}$ is strictly monotone. The sequence is also bounded (for example, by 2 , as it follows from Lemma 4 in Section 5); thus, it converges to a finite value. This fact proves that $\lambda_{\infty}=\lim _{\ell \rightarrow \infty} \lim _{r \rightarrow \infty} \Lambda_{r, \ell}=\lim _{\ell \rightarrow \infty} \lambda_{\ell}$ exists. Moreover, each $\lambda_{\ell}$ is strictly greater than 1 since $\lambda_{2}=1.184 \ldots$..

Equation (9b) says that $\boldsymbol{a}_{k}=S_{\ell}^{k-\ell} \boldsymbol{a}_{\ell}$ for every $k$, and (9a) that $\boldsymbol{a}_{\ell}$ is a nonnegative (and non-zero) vector: under these conditions, the primitivity of $S_{\ell}$ ensures (see [7], Th. 8.5.1) that the quotient $\boldsymbol{a}_{k} / \lambda_{\ell}^{k}$ converges to a positive vector. This means that we can write $a_{k, 1}=\left(\alpha_{\ell}+o(1)\right) \lambda_{\ell}^{k}$ for a suitable constant $\alpha_{\ell}>0$ as $k$ diverges. Based on the bound $\mathcal{W}(1, k) / k ! \geq a_{k, 1}$, we deduce that $\liminf _{k \rightarrow+\infty}(\mathcal{W}(1, k) / k !)^{1 / k} \geq \lambda_{\ell}$ for every $\ell$, so that:

$$
\liminf _{k \rightarrow+\infty}(\mathcal{W}(1, k) / k !)^{1 / k} \geq \lambda_{\infty}
$$

\section{Characteristic polynomials and computation of $\lambda_{\infty}$}

An elementary computation shows that the characteristic polynomial $p_{\ell}(x)$ of $S_{\ell}$ (which is a sparse $\ell^{2} \times \ell^{2}$ matrix) can be computed also as a determinant of an $\ell \times \ell$ matrix according to the following formula:

$$
p_{\ell}(x)=\operatorname{det}\left(x \mathbb{I}_{\ell^{2}}-S_{\ell}\right)=\operatorname{det}\left(x^{\ell} \mathbb{I}_{\ell}-\left(x^{\ell-1} A_{1}^{(\ell)}+x^{\ell-2} A_{2}^{(\ell)}+\cdots+x A_{\ell-1}^{(\ell)}+A_{\ell}^{(\ell)}\right)\right) \text {. }
$$

If we set

$$
\mathcal{A}_{\ell}(x):=x A_{1}^{(\ell)}+x^{2} A_{2}^{(\ell)}+\cdots+x^{\ell} A_{\ell}^{(\ell)}=\left(\begin{array}{ccccc}
\frac{x}{1 !} & \frac{x}{0 !} & 0 & \cdots & 0 \\
\frac{x^{2}}{3 !} & \frac{x^{2}}{2 !} & \frac{x^{2}}{1 !} & \cdots & 0 \\
\frac{x^{3}}{5 !} & \frac{x^{3}}{4 !} & \frac{x^{3}}{3 !} & \cdots & 0 \\
\ldots \ldots \ldots \ldots \ldots & \ldots \ldots \ldots \ldots \\
\frac{x^{\ell}}{(2 \ell-1) !} & \frac{x^{\ell}}{(2 \ell-2) !} & \frac{x^{\ell}}{(2 \ell-3) !} & \cdots & \frac{x^{\ell}}{\ell !}
\end{array}\right)
$$

and $q_{\ell}(x):=\operatorname{det}\left(\mathbb{I}_{\ell}-\mathcal{A}_{\ell}(x)\right)$, then $p_{\ell}(x)=x^{\ell^{2}} q_{\ell}(1 / x)$ and every non-zero root of $p_{\ell}(x)$ can be recovered as the inverse of a root of $q_{\ell}(x)$. Note that the degree of $q_{\ell}(x)$ is $\left(\begin{array}{c}\ell+1 \\ 2\end{array}\right)$. Some examples:

$$
\begin{aligned}
& q_{2}(x)=\frac{x^{3}}{3}-\frac{x^{2}}{2}-x+1, \\
& q_{3}(x)=-\frac{x^{6}}{45}+\frac{x^{5}}{24}+\frac{x^{4}}{6}+\frac{x^{3}}{6}-\frac{x^{2}}{2}-x+1,
\end{aligned}
$$




$$
\begin{aligned}
q_{4}(x)= & \frac{x^{10}}{4725}-\frac{x^{9}}{2160}-\frac{x^{8}}{360}-\frac{5 x^{7}}{504}-\frac{x^{6}}{360}+\frac{x^{5}}{12}+\frac{x^{4}}{8}+\frac{x^{3}}{6}-\frac{x^{2}}{2}-x+1, \\
q_{5}(x)= & -\frac{x^{15}}{4465125}+\frac{x^{14}}{1814400}+\frac{x^{13}}{226800}+\frac{19 x^{12}}{680400}+\frac{47 x^{11}}{518400}-\frac{107 x^{10}}{604800}-\frac{31 x^{9}}{20160}-\frac{11 x^{8}}{2520} \\
& -\frac{29 x^{7}}{5040}+\frac{x^{6}}{180}+\frac{3 x^{5}}{40}+\frac{x^{4}}{8}+\frac{x^{3}}{6}-\frac{x^{2}}{2}-x+1, \\
q_{6}(x) & =\frac{x^{21}}{46414974375}-\frac{x^{20}}{17146080000}-\frac{x^{19}}{1714608000}-\frac{101 x^{18}}{18860688000}-\frac{1703 x^{17}}{50295168000} \\
& -\frac{1613 x^{16}}{25147584000}+\frac{841 x^{15}}{1524096000}+\frac{97 x^{14}}{25401600}+\frac{827 x^{13}}{59875200}+\frac{169 x^{12}}{4989600}-\frac{x^{11}}{120960} \\
& -\frac{1307 x^{10}}{3628800}-\frac{643 x^{9}}{362880}-\frac{37 x^{8}}{10080}-\frac{11 x^{7}}{2520}+\frac{x^{6}}{240}+\frac{3 x^{5}}{40}+\frac{x^{4}}{8}+\frac{x^{3}}{6}-\frac{x^{2}}{2}-x+1 .
\end{aligned}
$$

The above examples show that $q_{\ell}(x)$ and $q_{\ell-1}(x)$ share the coefficients of the first $\ell$ powers and that these coefficients are the greatest ones in size; therefore, we expect that $\left|q_{\ell}(x)-q_{\ell-1}(x)\right|$ could be considerably smaller than $\left|q_{\ell}(x)\right|$ and $\left|q_{\ell-1}(x)\right|$. This is the content of Lemma 3 here below. We need the following auxiliary inequality that probably has some independent interest.

Lemma 2. Let $1 \leq a_{1} \leq a_{2} \leq \ldots \leq a_{k}$ be positive integers and let $a_{i, j}:=2 a_{i}-a_{j}$, for every $i, j \leq k$. Let $\sigma$ be an arbitrary but fixed permutation of $\{1, \ldots, k\}$ and suppose that $a_{\sigma(j), j} \geq 0$ for every $j$, then:

$$
\prod_{j=1}^{k} a_{\sigma(j), j} ! \geq \prod_{j=1}^{k} a_{j, j} !
$$

Proof. We prove the lemma by induction on $k$. For $k=1$ the claim is trivial, so we suppose $k>1$. The claim immediately follows by induction when $\sigma$ fixes $k$, hence we can further assume that $\sigma(k) \neq k$. We write the inequality as:

$$
\left[\prod_{\substack{j \neq k \\ j \neq \sigma^{-1}(k)}} a_{\sigma(j), j} !\right] \frac{a_{k, \sigma^{-1}(k)} !}{a_{\sigma^{-1}(k), \sigma^{-1}(k)} !} \geq\left[\prod_{\substack{j \neq k \\ j \neq \sigma^{-1}(k)}} a_{j, j} !\right] \frac{a_{k, k} !}{a_{\sigma(k), k} !},
$$

i.e.,

$$
\left[\prod_{\substack{j \neq k \\ j \neq \sigma^{-1}(k)}} a_{\sigma(j), j} !\right] \frac{\left(2 a_{k}-a_{\sigma^{-1}(k)}\right) !}{a_{\sigma^{-1}(k)} !} \geq\left[\prod_{\substack{j \neq k \\ j \neq \sigma^{-1}(k)}} a_{j, j} !\right] \frac{a_{k} !}{\left(2 a_{\sigma(k)}-a_{k}\right) !} .
$$

Since $2 a_{k}-a_{\sigma^{-1}(k)} \geq a_{\sigma^{-1}(k)}$ and $a_{k} \geq 2 a_{\sigma(k)}-a_{k}$, we can write the inequality as:

$$
\left[\prod_{\substack{j \neq k \\ j \neq \sigma^{-1}(k)}} a_{\sigma(j), j} !\right]\left(2 a_{k}-a_{\sigma^{-1}(k)}\right)_{\left(2 a_{k}-2 a_{\sigma^{-1}(k)}\right)} \geq\left[\prod_{\substack{j \neq k \\ j \neq \sigma^{-1}(k)}} a_{j, j} !\right]\left(a_{k}\right)_{\left(2 a_{k}-2 a_{\sigma(k)}\right)} .
$$

Note that $2 a_{k}-a_{\sigma^{-1}(k)} \geq a_{k}$, hence each factor of the form $2 a_{k}-a_{\sigma^{-1}(k)}-u$ coming from $\left(2 a_{k}-a_{\sigma^{-1}(k)}\right)_{\left(2 a_{k}-2 a_{\sigma^{-1}(k)}\right)}$ to LHS is not lower than the corresponding factor $a_{k}-u$ in $\left(a_{k}\right)_{\left(2 a_{k}-2 a_{\sigma(k)}\right)}$ to RHS, when $u \leq \min \left\{2 a_{k}-2 a_{\sigma^{-1}(k)}, 2 a_{k}-2 a_{\sigma(k)}\right\}$. There are three cases, according to the values of $\sigma^{-1}(k)$ and $\sigma(k)$.

Case 1: $\sigma^{-1}(k)=\sigma(k)$. Then there are as many factors in $\left(2 a_{k}-a_{\sigma^{-1}(k)}\right)_{\left(2 a_{k}-2 a_{\sigma^{-1}(k)}\right)}$ 
as in $\left(a_{k}\right)_{\left(2 a_{k}-2 a_{\sigma(k)}\right)}$ so that (13) is implied by the inequality:

$$
\prod_{\substack{j \neq k \\ j \neq \sigma^{-1}(k)}} a_{\sigma(j), j} ! \geq \prod_{\substack{j \neq k \\ j \neq \sigma^{-1}(k)}} a_{j, j} !
$$

Under the hypothesis $\sigma^{-1}(k)=\sigma(k)$ the restriction of $\sigma$ to the set $\{1, \ldots, k\} \backslash\left\{\sigma^{-1}(k)\right.$, $k\}$ is a permutation, hence (14) holds true by inductive hypothesis.

Case 2: $\sigma^{-1}(k)<\sigma(k)$. Then (13) is implied by the inequality:

$$
\left[\prod_{\substack{j \neq k \\ j \neq \sigma^{-1}(k)}} a_{\sigma(j), j} !\right]\left(2 a_{\sigma(k)}-a_{\sigma^{-1}(k)}\right)_{\left(2 a_{\sigma(k)}-2 a_{\sigma^{-1}(k)}\right)} \geq\left[\prod_{\substack{j \neq k \\ j \neq \sigma^{-1}(k)}} a_{j, j} !\right] .
$$

Since $\left(2 a_{\sigma(k)}-a_{\sigma^{-1}(k)}\right)_{\left(2 a_{\sigma(k)}-2 a_{\sigma^{-1}(k)}\right)}=\frac{a_{\sigma_{(k), \sigma^{-1}(k)}} !}{a_{\sigma^{-1}(k), \sigma^{-1}(k)}}$, this inequality is equivalent to:

$$
\left[\prod_{\substack{j \neq k \\ j \neq \sigma^{-1}(k)}} a_{\sigma(j), j} !\right] a_{\sigma(k), \sigma^{-1}(k)} ! \geq\left[\prod_{\substack{j \neq k \\ j \neq \sigma^{-1}(k)}} a_{j, j} !\right] a_{\sigma^{-1}(k), \sigma^{-1}(k)} ! .
$$

Case 3: $\sigma^{-1}(k)>\sigma(k)$. Then (13) is implied by the inequality:

$$
\left.\prod_{\substack{j \neq k \\ j \neq \sigma^{-1}(k)}} a_{\sigma(j), j} ! \geq\left[\prod_{\substack{j \neq k \\ j \neq \sigma^{-1}(k)}} a_{j, j} !\right]\left(2 a_{\sigma^{-1}(k)}-a_{k}\right)_{\left(2 a_{\sigma^{-1}(k)}\right.}-2 a_{\sigma(k)}\right) .
$$

We note that $\left(2 a_{\sigma^{-1}(k)}-a_{k}\right)_{\left(2 a_{\sigma^{-1}(k)}-2 a_{\sigma(k)}\right)}=\frac{a_{\sigma^{-1}(k), k} !}{a_{\sigma(k), k} !}$, where $a_{\sigma^{-1}(k), k}$ is nonnegative because the assumption $\sigma^{-1}(k)>\sigma(k)$ implies that $a_{\sigma^{-1}(k), k} \geq a_{\sigma(k), k}$. Also $a_{\sigma(k), \sigma^{-1}(k)}$ is nonnegative, because $a_{\sigma(k), \sigma^{-1}(k)} \geq a_{\sigma(k), k}$. We prove now that:

$$
\frac{a_{\sigma^{-1}(k), k} !}{a_{\sigma(k), k} !} \leq \frac{a_{\sigma^{-1}(k), \sigma^{-1}(k)} !}{a_{\sigma(k), \sigma^{-1}(k)} !} .
$$

In fact, in terms of the original sequence $a_{j}$ the inequality in (17) means that:

$$
\frac{\left(2 a_{\sigma^{-1}(k)}-a_{k}\right) !}{\left(2 a_{\sigma(k)}-a_{k}\right) !} \leq \frac{a_{\sigma^{-1}(k)} !}{\left(2 a_{\sigma(k)}-a_{\sigma^{-1}(k)}\right) !} .
$$

Under the condition $\sigma(k)<\sigma^{-1}(k)<k$ it becomes:

$$
\left.\left(2 a_{\sigma^{-1}(k)}-a_{k}\right)_{\left(2 a_{\sigma^{-1}(k)}\right.}-2 a_{\sigma}(k)\right) \leq\left(a_{\sigma^{-1}(k)}\right)_{\left(2 a_{\sigma^{-1}(k)}-2 a_{\sigma(k)}\right)}
$$

which is evident, because $2 a_{\sigma^{-1}(k)}-a_{k} \leq a_{\sigma^{-1}(k)}$. Concluding, substituting (17) in (16) we see that also in this case the original inequality (13) is implied by (15). This means that by proving (15) we prove both Case 2 and Case 3. To this purpose we define $\tilde{\sigma}(j)$ for $j=1, \ldots, k-1$ as follows: $\tilde{\sigma}(j):=\sigma(j)$ if $j \neq \sigma^{-1}(k)$, and $\tilde{\sigma}\left(\sigma^{-1}(k)\right):=\sigma(k)$. Then $\tilde{\sigma}$ is a permutation of $\{1, \ldots, k-1\}$ and (15) can be written as:

$$
\prod_{j \neq k} a_{\tilde{\sigma}(j), j} ! \geq \prod_{j \neq k} a_{j, j} !
$$

This inequality holds true, by inductive hypothesis, since it involves only $k-1$ numbers. 
Lemma 3. For every $\ell>2$ and every $x$ we have:

$$
\left|q_{\ell}(x)-q_{\ell-1}(x)\right| \leq \frac{e|x|^{\ell}}{\ell !}\left[1+\sum_{n=1}^{+\infty} \frac{\lfloor\sqrt{2 n}\rfloor^{n+1}}{n !}|x|^{n}\right] .
$$

In particular,

$$
\max _{|x| \leq 1}\left|q_{\ell}(x)-q_{\ell-1}(x)\right| \leq \frac{e C}{\ell !}
$$

where $C:=1+\sum_{n=1}^{+\infty}\lfloor\sqrt{2 n}\rfloor^{n+1} / n !=33.15 \ldots$, so that:

$$
\max _{|x| \leq 1}\left|q_{\ell}(x)-q_{6}(x)\right| \leq e C \sum_{u=7}^{+\infty} \frac{1}{u !} \leq \frac{e^{2} C}{7 !} \leq 0.05 \quad \forall \ell \geq 6 .
$$

Note that (18) confirms the correctness of our previous remark about the equality of the coefficients of $x^{j}$ with $j<\ell$ in $q_{\ell-1}(x)$ and $q_{\ell}(x)$.

Proof. Consider the representation of $q_{\ell}(x)$ as determinant of the matrix $\mathbb{I}_{\ell}-\mathcal{A}_{\ell}(x)$ (see (12)), that we compute using the Laplace formula with respect to the last line, obtaining:

$$
q_{\ell}(x)=\sum_{m=1}^{\ell}(-1)^{\ell+m}\left(\delta_{m=\ell}-\frac{x^{\ell}}{(2 \ell-m) !}\right) \cdot T_{\ell, m}(x),
$$

where $T_{\ell, m}(x)$ denotes the polynomial which is the $\ell, m$ cofactor. In this formula $T_{\ell, \ell}(x)$ is the cofactor of the lower-right entry, hence it is equal to the determinant of $\mathbb{I}_{\ell-1}-\mathcal{A}_{\ell-1}(x)$ and therefore coincides with $q_{\ell-1}(x)$. It follows that:

$$
q_{\ell}(x)-q_{\ell-1}(x)=-\sum_{m=1}^{\ell}(-1)^{\ell+m} \frac{x^{\ell}}{(2 \ell-m) !} T_{\ell, m}(x)
$$

implying that:

$$
\left|q_{\ell}(x)-q_{\ell-1}(x)\right| \leq|x|^{\ell} \sum_{m=1}^{\ell} \frac{1}{(2 \ell-m) !}\left|T_{\ell, m}(x)\right| .
$$

To complete the proof we need a bound for $\left|T_{\ell, m}(x)\right|$. For every positive integer $n$ let $\mathcal{P}(n)$ be the set of partitions of $n$ in distinct parts and for every $\pi \in \mathcal{P}(n)$, let $M_{\pi}$ be the submatrix of $-\mathcal{A}_{\ell}(1)$ that we obtain by intersecting the rows and the columns whose indexes are in $\pi$. For example,

$$
\pi=(4,5,7) \quad \Longrightarrow \quad M_{\pi}=-\left(\begin{array}{ccc}
1 / 4 ! & 1 / 3 ! & 1 / 1 ! \\
1 / 6 ! & 1 / 5 ! & 1 / 3 ! \\
1 / 10 ! & 1 / 9 ! & 1 / 7 !
\end{array}\right)
$$

It is easy to verify that:

$$
T_{\ell, m}(x)=\delta_{m=\ell}+\sum_{n=1}^{\left(\begin{array}{l}
\ell \\
2
\end{array}\right)}\left[\sum_{\pi \in \mathcal{P}(n)}^{*} \operatorname{det} M_{\pi}\right] x^{n}
$$


where $\sum^{*}$ means that the sum is restricted to those partitions $\pi$ which do not contain $m$. We bound this sum trivially as:

$$
\left|T_{\ell, m}(x)\right| \leq 1+\sum_{n=1}^{\left(\begin{array}{c}
\ell \\
2
\end{array}\right)}\left[\sum_{\pi \in \mathcal{P}(n)}\left|\operatorname{det} M_{\pi}\right|\right]|x|^{n}
$$

where now the inner sum is extended to the whole set of partitions in distinct parts (not only to those ones avoiding $m$ ). In particular, RHS in (23) is independent of $m$.

Let $a_{1}<a_{2}<\cdots<a_{k}$ be the sequence describing $\pi$ : each entry $m_{i, j}$ of $-M_{\pi}$ is equal to $1 / a_{i, j}$ ! where $a_{i, j}=2 a_{i}-a_{j}$ and where $1 / a_{i, j}$ ! is set to 0 when $a_{i, j}<0$. By Lemma 2, each product $\prod_{j=1}^{k} m_{\sigma(j), j}$ which is not zero is not greater than the product of the terms coming from the main diagonal. Hence the determinant of $M_{\pi}$ is bounded by $k ! / a_{1} ! \cdots a_{k} !$, so that:

$$
\sum_{\pi \in \mathcal{P}(n)}\left|\operatorname{det} M_{\pi}\right| \leq \sum_{k=1} \sum_{\substack{a_{1}, \ldots, a_{k} \\ a_{1}<\cdots<a_{k} \\ a_{1}+\cdots+a_{k}=n}} \frac{k !}{a_{1} ! \cdots a_{k} !} .
$$

The strict inequality $a_{1}<\cdots<a_{k}$ forces $k$ to be lower than $\sqrt{2 n}$, therefore:

$$
\sum_{\pi \in \mathcal{P}(n)}\left|\operatorname{det} M_{\pi}\right| \leq \sum_{k=1}^{\sqrt{2 n}} \frac{k !}{n !} \sum_{\begin{array}{c}
a_{1}, \ldots, a_{k} \\
a_{1}<\cdots<a_{k} \\
a_{1}+\cdots+a_{k}=n
\end{array}} \frac{n !}{a_{1} ! \cdots a_{k} !} .
$$

The factor $k$ ! can be included in the inner sum by substituting the prescription $a_{1}<\cdots<a_{k}$ with the unordered one: $a_{u} \neq a_{v}$ for every $u \neq v$. The resulting sum can be bounded by $\sum_{\substack{a_{1}, \ldots, a_{k} \geq 0 \\ a_{1}+\cdots+a_{k}=n}} \frac{n !}{a_{1} ! \cdots a_{k} !}$ whose value is $k^{n}$, therefore we have proved that:

$$
\sum_{\pi \in \mathcal{P}(n)}\left|\operatorname{det} M_{\pi}\right| \leq \sum_{k=1}^{\sqrt{2 n}} \frac{k^{n}}{n !} \leq \frac{\lfloor\sqrt{2 n}\rfloor^{n+1}}{n !} .
$$

Substituting this inequality in (23) and then in (22) we conclude that:

$$
\left|q_{\ell}(x)-q_{\ell-1}(x)\right| \leq|x|^{\ell} \sum_{m=1}^{\ell} \frac{1}{(2 \ell-m) !}\left[1+\sum_{n=1}^{\left(\begin{array}{c}
\ell \\
2
\end{array}\right)} \frac{\lfloor\sqrt{2 n}\rfloor^{n+1}}{n !}|x|^{n}\right],
$$

which gives (18), because $\sum_{m=1}^{\ell} \frac{1}{(2 \ell-m) !} \leq \frac{e}{\ell !}$.

Remark. The last estimations proving Lemma 3 are not optimal and could easily be improved, although at the cost of some complications in the presentation of the final result.

Now we show how to estimate the difference $\left|\lambda_{\ell}^{-1}-\lambda_{\infty}^{-1}\right|$; in this way, we will be able to compute the value of $\lambda_{\infty}$ with arbitrary precision (see Formula (24) below). This is an important point in our proof: the value of $\lambda_{\infty}$ will appear in several explicit inequalities in the next section devoted to the proof of the equality of $\lambda_{\infty}$ and $\Lambda_{\infty}$. Some of them need computations that we refer to a software but are possible only as a consequence of our ability to detect both the value of $\lambda_{\infty}$ and the 
rate of convergence of $\lambda_{\ell}$ to $\lambda_{\infty}$.

We know that $\lambda_{\ell}^{-1}$ is a root of $q_{\ell}(x)$ in $(0,1)$; therefore:

$$
\left|q_{\ell}\left(\lambda_{\ell-1}^{-1}\right)-q_{\ell-1}\left(\lambda_{\ell-1}^{-1}\right)\right|=\left|q_{\ell}\left(\lambda_{\ell-1}^{-1}\right)-q_{\ell}\left(\lambda_{\ell}^{-1}\right)\right|=\left|\lambda_{\ell-1}^{-1}-\lambda_{\ell}^{-1}\right| \cdot\left|q_{\ell}^{\prime}(\zeta)\right|,
$$

where $\zeta \in\left(\lambda_{\ell}^{-1}, \lambda_{\ell-1}^{-1}\right) \subset(0,1)$. A bound for $\left|\lambda_{\ell-1}^{-1}-\lambda_{\ell}^{-1}\right|$ will come from an upper bound for $\left|q_{\ell}(x)-q_{\ell-1}(x)\right|$ and a lower bound for $\left|q_{\ell}^{\prime}(x)\right|$, both in $(0,1)$. The upper bound is provided by Lemma 3: suppose we have already computed $\lambda_{\ell^{\prime}}^{-1}$; then we know that $\lambda_{\ell}^{-1}<\lambda_{\ell^{\prime}}^{-1}$ for every $\ell>\ell^{\prime}$, and from (18) we have:

$$
\left|q_{\ell}\left(\lambda_{\ell-1}^{-1}\right)-q_{\ell-1}\left(\lambda_{\ell-1}^{-1}\right)\right| \leq \frac{e \lambda_{\ell^{\prime}}^{-\ell}}{\ell !}\left[1+\sum_{n=1}^{+\infty} \frac{\lfloor\sqrt{2 n}\rfloor^{n+1}}{n !} \lambda_{\ell^{\prime}}^{-n}\right] \quad \forall \ell>\ell^{\prime} .
$$

Starting with (21) and using the same argument proving (18), we get that:

$$
\left|q_{u}^{\prime}(x)-q_{u-1}^{\prime}(x)\right| \leq \frac{e|x|^{u-1}}{u !}\left[u+\sum_{n=1}^{+\infty}(u+n) \frac{\lfloor\sqrt{2 n}\rfloor^{n+1}}{n !}|x|^{n}\right] \quad \forall u,
$$

so that in $|x| \leq 1$ we have:

$$
\left|q_{u}^{\prime}(x)-q_{u-1}^{\prime}(x)\right| \leq \frac{e}{u !}\left[u+\sum_{n=1}^{+\infty}(u+n) \frac{\lfloor\sqrt{2 n}\rfloor^{n+1}}{n !}\right] \quad \forall u .
$$

Adding this inequality for $u=\ell^{\prime}+1, \ldots, \ell$ we get:

$$
\max _{|x| \leq 1}\left|q_{\ell}^{\prime}(x)-q_{\ell^{\prime}}^{\prime}(x)\right| \leq \frac{e^{2} C}{\ell^{\prime} !}+\frac{e^{2} D}{\left(\ell^{\prime}+1\right) !}
$$

where $C$ is the constant appearing in Lemma 3 and $D:=\sum_{n=1}^{+\infty}\lfloor\sqrt{2 n}\rfloor^{n+1} /(n-1) !=$ $199.64 \ldots$... This formula proves that:

$$
\left|q_{\ell}^{\prime}(x)-q_{10}^{\prime}(x)\right| \leq 0.001
$$

in $|x| \leq 1$, for every $\ell \geq 10$. Since $\min _{x \in(0,1)}\left|q_{10}^{\prime}(x)\right| \geq 0.678$ (a fact which is proved using PARIgp [13] for the necessary computations), we obtain that $\left|q_{\ell}^{\prime}(x)\right| \geq 0.677$ in $(0,1)$ for every $\ell \geq 10$. Concluding, we have proved that:

$$
\left|\lambda_{\ell-1}^{-1}-\lambda_{\ell}^{-1}\right| \leq \frac{e}{0.677} \frac{\lambda_{\ell^{\prime}}^{-\ell}}{\ell !}\left[1+\sum_{n=1}^{+\infty} \frac{\lfloor\sqrt{2 n}\rfloor^{n+1}}{n !} \lambda_{\ell^{\prime}}^{-n}\right] \quad \forall \ell>\ell^{\prime} \geq 10
$$

and adding these inequalities we finally obtain that:

$$
\begin{aligned}
\left|\lambda_{\ell}^{-1}-\lambda_{\infty}^{-1}\right| & \leq \frac{e}{0.677} \sum_{u=\ell+1}^{+\infty} \frac{\lambda_{\ell^{\prime}}^{-u}}{u !}\left[1+\sum_{n=1}^{+\infty} \frac{\lfloor\sqrt{2 n}\rfloor^{n+1}}{n !} \lambda_{\ell^{\prime}}^{-n}\right] \\
& \leq \frac{e}{0.677} \frac{\lambda_{\ell^{\prime}}^{-\ell-1} e^{1 / \lambda_{\ell^{\prime}}}}{(\ell+1) !}\left[1+\sum_{n=1}^{+\infty} \frac{\lfloor\sqrt{2 n}\rfloor^{n+1}}{n !} \lambda_{\ell^{\prime}}^{-n}\right] \quad \forall \ell>\ell^{\prime} \geq 10 .
\end{aligned}
$$

A preliminary computation shows that $\lambda_{10}^{-1}<0.839$, hence the previous formula with $\ell^{\prime}=10$ and $\ell=30$ gives $\left|\lambda_{30}^{-1}-\lambda_{\infty}^{-1}\right| \leq 6.6 \cdot 10^{-35}$. Computing $\lambda_{30}^{-1}$ and recalling that $\lambda_{\infty}^{-1}<\lambda_{30}^{-1}$ we finally have:

$$
\lambda_{\infty}=1.192674341213466032221288982528755 \ldots
$$

with thirty four correct digits. 
Remark. Since the minimum value for $\left|q_{6}(x)\right|$ in $|x|=1$ is reached at $x=1$ and is $0.13 \ldots$, based on (20) it follows that:

$$
\left|q_{\ell}(x)-q_{6}(x)\right|<\left|q_{6}(x)\right| \quad|x|=1
$$

for every $\ell \geq 6$. By Rouché's theorem, this proves that each polynomial $q_{\ell}(x)$ with $\ell \geq 6$ has a unique root in $|x| \leq 1$ since this is what occurs to $q_{6}(x)$; this implies that each eigenvalue of $S_{\ell}$ that is not $\lambda_{\ell}$ is strictly lower than 1 in absolute value. This fact and the value of $\lambda_{\infty}$ we have just computed show that there is a uniform (i.e., independent of $\ell$ ) gap between the maximal eigenvalue $\lambda_{\ell}$ of $S_{\ell}$ and the other eigenvalues. This fact is not an essential part of our argument, but later we will introduce a new family of matrices that are strictly related to the $S_{\ell}$ matrices: the existence of a gap in the eigenvalues of also these matrices (see Lemma 10) will be fundamental for the conclusion of the proof of the theorem.

\section{UpPer Bound AND EQUALITy of $\lambda_{\infty}$ AND $\Lambda_{\infty}$}

We start with a simple but important remark: in each column of $S_{\ell}$ there are two non-zero entries, at most; in the first and the second column these entries are equal to 1 , while in the other columns one entry is not greater than 1 and the second one (if present) is equal to 1 . Hence, we have proved that:

Lemma 4. $\Lambda_{1, \ell}=\left\|S_{\ell}\right\|=2$ for every $\ell$.

The number 2 is not an eigenvalue of $S_{\ell}$ because each $\lambda_{\ell}$ is bounded by $\lambda_{\infty}$, whose value is $1.19 \ldots$ Hence $(10 \mathrm{~b})$ has the solution $\boldsymbol{w}_{k}=2^{k} \boldsymbol{w}$, where $\boldsymbol{w}$ is $\frac{1}{(\ell+1) ! !^{2}}\left[\mathbb{I}_{\ell^{2}}-\right.$ $\left.\frac{1}{2} S_{\ell}\right]^{-1} \boldsymbol{z}$. We need a bound, uniform in $\ell$, for the size of $\boldsymbol{w}$, but the definition of $\boldsymbol{w}$ in terms of $S_{\ell}$ is not suitable for this purpose as a consequence of the previous lemma. We can overcome this difficulty by exploiting the special structure of $S_{\ell}$ in the following way. For each vector $\boldsymbol{y} \in \mathbb{C}^{\ell^{2}}$, let $\boldsymbol{y}^{\text {red }}$ in $\mathbb{C}^{\ell}$ be the projection of $\boldsymbol{y}$ along the first $\ell$ coordinates. The last $\ell^{2}-\ell$ entries in $z$ are null and the last $\ell^{2}-\ell$ rows of $S_{\ell}$ are $\left(\mathbb{I}_{\ell^{2}-\ell}, O_{\left(\ell^{2}-\ell\right) \times \ell}\right)$; hence, $\boldsymbol{w}$ has the form:

$$
\boldsymbol{w}=\left(\begin{array}{c}
\boldsymbol{w}^{\mathrm{red}} \\
\boldsymbol{w}^{\mathrm{red}} / 2 \\
\cdots \\
\boldsymbol{w}^{\mathrm{red}} / 2^{\ell-1}
\end{array}\right)
$$

where $\boldsymbol{w}^{\text {red }}$ satisfies the reduced system:

$$
\boldsymbol{w}^{\mathrm{red}}=\mathcal{A}_{\ell}\left(\frac{1}{2}\right) \boldsymbol{w}^{\mathrm{red}}+\frac{1}{(\ell+1) !^{2}} \boldsymbol{z}^{\mathrm{red}}
$$

and therefore:

$$
\boldsymbol{w}^{\mathrm{red}}=\frac{1}{(\ell+1) !^{2}}\left[\mathbb{I}_{\ell}-\mathcal{A}_{\ell}\left(\frac{1}{2}\right)\right]^{-1} \boldsymbol{z}^{\mathrm{red}} .
$$

In this way, $\boldsymbol{w}$ has been related to the inverse of a different matrix, and this new relation allows us to prove the following bound.

Lemma 5. $\left\|\boldsymbol{w}_{k}\right\| \ll \frac{2^{k}}{\ell !^{2}}$, uniformly in $k$ and $\ell$.

Proof. By (25) it is sufficient to prove that $\left\|\boldsymbol{w}^{\text {red }}\right\| \ll 1 / \ell !^{2}$ uniformly in $\ell$. The column in $\mathcal{A}_{\ell}(x)$ having the greatest norm is the second one (this is evident from (12)) 
whose norm is $\sum_{s=0}^{\ell-1}|x|^{s+1} /(2 s)$ !. Hence $\left\|\mathcal{A}_{\ell}(1 / 2)\right\| \leq \mathrm{Ch}(1 / \sqrt{2}) / 2<1$, independently of $\ell$. As a consequence, $\left(\mathbb{I}_{\ell}-\mathcal{A}_{\ell}\left(\frac{1}{2}\right)\right)^{-1}$ is given by the usual power series in $\mathcal{A}_{\ell}\left(\frac{1}{2}\right)$ and

$$
\left\|\left(\mathbb{I}_{\ell}-\mathcal{A}_{\ell}\left(\frac{1}{2}\right)\right)^{-1}\right\| \leq \frac{1}{1-\left\|\mathcal{A}_{\ell}\left(\frac{1}{2}\right)\right\|} \leq \frac{2}{2-\operatorname{Ch}(1 / \sqrt{2})} \quad \forall \ell .
$$

Therefore,

$$
\left\|\boldsymbol{w}^{\mathrm{red}}\right\| \leq \frac{1}{(\ell+1) !^{2}}\left\|\left(\mathbb{I}_{\ell}-\mathcal{A}_{\ell}\left(\frac{1}{2}\right)\right)^{-1}\right\| \cdot\left\|\boldsymbol{z}^{\mathrm{red}}\right\| \ll \frac{1}{\ell !^{2}} .
$$

The difference $\boldsymbol{v}_{k}:=\boldsymbol{b}_{k}-\boldsymbol{w}_{k}$ satisfies the homogeneous system:

$$
\boldsymbol{v}_{\ell}:=\boldsymbol{b}_{\ell}-\boldsymbol{w}_{\ell}, \quad \boldsymbol{v}_{k}=S_{\ell} \boldsymbol{v}_{k-1} \quad \forall k>\ell
$$

therefore $\boldsymbol{v}_{k}=S_{\ell}^{k-\ell} \boldsymbol{v}_{\ell}$. The vector $\boldsymbol{b}_{\ell}$ is defined by the entries of $N_{k, l}$ for $k, l \leq \ell$ (see (10a)); hence, $\left\|\boldsymbol{b}_{\ell}\right\| \ll 2^{\ell}$ uniformly in $\ell$ in accordance with Lemma 1; this bound and Lemma 5 give for $\boldsymbol{v}_{\ell}$ the bound $\left\|\boldsymbol{v}_{\ell}\right\| \ll 2^{\ell}$. We need an analogous upper bound for $\left\|\boldsymbol{v}_{k}\right\|$ when $k>\ell$. The norm of $S_{\ell}$ is larger than its spectral radius $\lambda_{\ell}$, so there is no possibility to prove that $\left\|\boldsymbol{v}_{k}\right\| \ll \lambda_{\ell}^{k-\ell} 2^{\ell}$. However, for every positive integer $r$, we have the identity $\boldsymbol{v}_{k}=S_{\ell}^{k-\ell} \boldsymbol{v}_{\ell}=\left(S_{\ell}^{r}\right)^{\lfloor(k-\ell) / r\rfloor}\left(S_{\ell}\right)^{r[(k-\ell) / r-\lfloor(k-\ell) / r\rfloor]} \boldsymbol{v}_{\ell}$; recalling that $\Lambda_{r, \ell}=\left\|S_{\ell}^{r}\right\|^{1 / r} \geq 1$ and according to Lemma 4 , we deduce that $\left\|\boldsymbol{v}_{k}\right\| \ll \Lambda_{r, \ell}^{k-\ell} 2^{\ell+r}$.

Adding the bounds for $\boldsymbol{v}_{k}$ and for $\boldsymbol{w}_{k}$, we obtain for the solution $\boldsymbol{b}_{k}$ of the original system the bound $\Lambda_{r, \ell}^{k-\ell} 2^{\ell+r}+\frac{2^{k}}{\ell !^{2}}$, uniformly in $k$ and $\ell$; thus, there exists a positive constant $\alpha$ such that:

producing the bound:

$$
b_{k, 1} \leq \alpha\left[\Lambda_{r, \ell}^{k-\ell} 2^{\ell+r}+\frac{2^{k}}{\ell !^{2}}\right]
$$

$$
\left(b_{k, 1}\right)^{1 / k} \leq \alpha^{1 / k} \Lambda_{r, \ell}\left[2^{\ell+r}+\frac{2^{k}}{\ell !^{2}}\right]^{1 / k} .
$$

We do not obtain any useful bound if we keep $r$ and $\ell$ fixed in (26) when $k$ diverges, and some kind of uniformity in these parameters must be exploited.

We know that $S_{\ell-1} \leq S_{\ell}$; hence, $\left\|S_{\ell-1}^{r}\right\| \leq\left\|S_{\ell}^{r}\right\|$ for every $\ell$ and $r$ : according to our notation, this means that $\Lambda_{r, \ell-1} \leq \Lambda_{r, \ell}$. Moreover, $\Lambda_{r, \ell} \leq \Lambda_{1, \ell}=2$ for every $r$ and $\ell$; hence, $\lim _{\ell \rightarrow \infty} \Lambda_{r, \ell}$ exists for every fixed $r$ : we denote it by $\Lambda_{r, \infty}$. We take $\ell=\left\lfloor k /\left(2 \log _{2} k\right)\right\rfloor$ in $(26)$. Then, $2^{\ell} \leq 2^{k /\left(2 \log _{2} k\right)}$ and $2^{k} / \ell !^{2} \ll 2^{k \log \log k / \log k}$ so that, by taking the limit $k \rightarrow \infty$, we conclude that

$$
\limsup _{k \rightarrow \infty}\left(b_{k, 1}\right)^{1 / k} \leq \Lambda_{r, \infty} \quad \forall r .
$$

LHS in (27) is independent of $r$; hence, we look for that value of $r$ giving the better upper bound. The following lemma shows that an optimal $r$ does not exist and we get better bounds as $r$ is larger.

Lemma 6. The limit $\Lambda_{\infty}=\lim _{r \rightarrow \infty} \Lambda_{r, \infty}$ exists and is lower than each $\Lambda_{r, \infty}$.

Proof. For $\ell \geq 2$, let $\mathcal{M}_{\ell}$ be the set of matrices $\ell^{2} \times \ell^{2}$, considered as Banach space with respect to the norm $\|\cdot\|$. Let $\mathcal{M}$ be the subset of sequences $\left(M_{2}, M_{3}, \ldots\right)$ in $\oplus_{\ell} \mathcal{M}_{\ell}$ for which $\sup _{\ell}\left\|M_{\ell}\right\|$ is finite. This set is an algebra with respect to the pointwise product and sum and becomes a Banach algebra when we introduce the norm $\left\|\left(M_{2}, M_{3}, \ldots\right)\right\|:=\sup _{\ell}\left\|M_{\ell}\right\|$. According to Lemma 4, the collection $\left(S_{2}, S_{3}, \ldots\right)$ 
defines an element in $\mathcal{M}$. The norm (in $\mathcal{M}$ ) of the $r$ th power of $\left(S_{2}, S_{3}, \ldots\right)$ is $\sup _{\ell}\left\|S_{\ell}^{r}\right\|$, and this number is $\Lambda_{r, \infty}^{r}$ (because $\left\|S_{\ell}^{r}\right\|=\Lambda_{r, \ell}^{r}$ and $\Lambda_{r, \ell-1} \leq \Lambda_{r, \ell}$ for every $r$ and $\ell$ ). Then, the existence of the limit of $\Lambda_{r, \infty}$ as $r$ diverges is now a direct consequence of Gelfand's formula for the spectral radius of the element $\left(S_{2}, S_{3}, \ldots\right)$ in $\mathcal{M}$ (see [18], Th. 18.9). This fact also proves that $\Lambda_{\infty} \leq \Lambda_{r, \infty}$ for every $r$.

Lemma 6 and (27) give the upper bound:

$$
\limsup _{k \rightarrow \infty}(\mathcal{W}(1, k) / k !)^{1 / k} \leq \Lambda_{\infty}
$$

Given (11) and (28), we will prove the theorem by proving that $\Lambda_{\infty}$ and $\lambda_{\infty}$ are equal. The construction we have used to prove Lemma 6 suggests a possible way to reach this goal: by definition, $\lambda_{\infty}$ is not lower than every spectral radius $\lambda_{\ell}$; hence, if $\lambda \in \mathbb{C}$ is fixed with $|\lambda|>\lambda_{\infty}$, then every matrix $\left(\lambda \mathbb{I}_{\ell^{2}}-S_{\ell}\right)^{-1}$ exists in $\mathcal{M}_{\ell}$. However, the sequence $\left(\left(\lambda \mathbb{I}_{2^{2}}-S_{2}\right)^{-1},\left(\lambda \mathbb{I}_{3^{2}}-S_{3}\right)^{-1}, \ldots\right)$ defines an element in $\mathcal{M}$ if and only if the norms $\left\|\left(\lambda \mathbb{I}_{\ell^{2}}-S_{\ell}\right)^{-1}\right\|$ are bounded. If we can prove this fact, then we can also conclude that $\Lambda_{\infty}$, the spectral radius in $\mathcal{M}$ of $\left(S_{2}, S_{3}, \ldots\right)$, is not greater than $|\lambda|$. Given the arbitrariness of $\lambda$, this means that $\Lambda_{\infty} \leq \lambda_{\infty}$ (and, hence, that $\Lambda_{\infty}=\lambda_{\infty}$ because the inequality $\Lambda_{\infty} \geq \lambda_{\infty}$ is evident). The argument can be easily reversed, thus proving that $\Lambda_{\infty}$ equals $\lambda_{\infty}$ if and only if $\left\|\left(\lambda \mathbb{I}_{\ell^{2}}-S_{\ell}\right)^{-1}\right\|$ is bounded in $\ell$, for every $\lambda$ with $|\lambda|>\lambda_{\infty}$. However, we have no simple argument proving that those norms are really bounded, and the proof of the equality of $\Lambda_{\infty}$ with $\lambda_{\infty}$ proceeds in a different way.

The following proposition shows that the value of $\left\|S_{\ell}^{r}\right\|$ grows with $\ell$ but becomes constant for $\ell \geq 2 r$ for every fixed $r$ (greater than 22, but only as a consequence of some technical assumptions); this claim is essentially a generalization of the previous Lemma 4. An explicit bound for $\Lambda_{r, \infty}$ is deduced.

Proposition 2. Let $d_{i, u}$ for $i \geq 0$ and $u \geq 1$ be defined as:

$$
d_{i, u}=0 \quad \text { if } u>2 i, \quad d_{i, u}=\sum_{\kappa=\lceil u / 2\rceil}^{i} \frac{1}{(2 \kappa-u) !}\left(1+d_{i-\kappa, \kappa}\right) \quad \text { if } u \leq 2 i .
$$

Then, for every $r \geq 22$

$$
\Lambda_{r, \infty}^{r}=\left\|S_{2 r}^{r}\right\|=1+\max _{j \leq r}\left\{\sum_{\kappa=0}^{r-1} \frac{1}{(2 \kappa) !}\left(1+d_{r-1-\kappa, j+\kappa}\right)\right\} .
$$

Moreover, we have the bound:

$$
\Lambda_{r, \infty}^{r}=\left\|S_{2 r}^{r}\right\| \leq 1+\operatorname{Ch}(1)\left(1+\max _{j<2 r} d_{r-1, j}\right) .
$$

With a bit of extra work it is possible to prove that the range for $j$ in (31) can be restricted to the even integers lower than $r / 2$; moreover, our computations show that for $r \leq 3 \cdot 10^{4}$ the maximum is actually attained at $j=2$, but we have not been able to prove that this is true in general.

Proof. We split the proof into several steps; the first ones (1-7) prove that the norm of $S_{\ell}^{r}$ is independent of $\ell$ when $\ell \geq 2 r$ so that $\Lambda_{r, \infty}^{r}=\left\|S_{2 r}^{r}\right\|$, while the last step proves formula (30) giving $\left\|S_{2 r}^{r}\right\|$ in terms of the sequence $d_{i, u}$. 
Step 1. Let $\ell$ be fixed and let $A_{r, j}^{(\ell)}$ be the double sequence of square matrices of order $\ell$ defined recursively as:

$$
A_{1, j}^{(\ell)}:=\left\{\begin{array}{ll}
A_{j}^{(\ell)} & \text { if } j \leq \ell \\
O_{\ell \times \ell} & \text { if } j>\ell
\end{array}, \quad A_{r+1, j}^{(\ell)}:=A_{r, 1}^{(\ell)} A_{1, j}^{(\ell)}+A_{r, j+1}^{(\ell)} .\right.
$$

Then, for $\ell>r$ we have:

$$
S_{\ell}^{r}=\left(\begin{array}{cccc}
A_{r, 1}^{(\ell)} & A_{r, 2}^{(\ell)} & \ldots & A_{r, \ell}^{(\ell)} \\
\ldots \ldots \ldots \ldots \ldots \ldots \ldots \ldots \ldots \ldots & \ldots \ldots \ldots \\
A_{2,1}^{(\ell)} & A_{2,2}^{(\ell)} & \ldots & A_{2, \ell}^{(\ell)} \\
A_{1,1}^{(\ell)} & A_{1,2}^{(\ell)} & \cdots & A_{1, \ell}^{(\ell)} \\
& \mathbb{I}_{(\ell-r) \ell} & & O_{(\ell-r) \ell \times r \ell}
\end{array}\right) .
$$

This fact is evident when $r=1$ and can be proved by induction on $r$ using the decomposition of $S_{\ell}^{r+1}$ as $S_{\ell}^{r} S_{\ell}$.

Let $B_{0, j}^{(\ell)}:=O_{\ell \times \ell}$ for every $j$, and $B_{r, j}^{(\ell)}:=\sum_{k=1}^{r} A_{k, j}^{(\ell)}$ when $r \geq 1$. Then:

$B_{r, j}^{(\ell)}=\sum_{k=1}^{r} A_{k, j}^{(\ell)}=A_{1, j}^{(\ell)}+\sum_{k=2}^{r} A_{k, j}^{(\ell)}=A_{1, j}^{(\ell)}+\sum_{k=1}^{r-1} A_{k, 1}^{(\ell)} A_{1, j}^{(\ell)}+\sum_{k=1}^{r-1} A_{k, j+1}^{(\ell)}$,

thus

$$
B_{r, j}^{(\ell)}=\left(\mathbb{I}_{\ell}+B_{r-1,1}^{(\ell)}\right) A_{1, j}^{(\ell)}+B_{r-1, j+1}^{(\ell)} .
$$

Iterating this equality we get also that:

$$
B_{r, j}^{(\ell)}=\sum_{k=1}^{r}\left(\mathbb{I}_{\ell}+B_{r-k, 1}^{(\ell)}\right) A_{1, j+k-1}^{(\ell)} .
$$

The interest for the matrices $B_{r, j}^{(\ell)}$ comes from the identity:

$$
\left\|S_{\ell}^{r}\right\|=\max \{\alpha, \beta\}, \quad \text { where }\left\{\begin{array}{l}
\alpha:=1+\max _{j \leq \ell-r}\left\|B_{r, j}^{(\ell)}\right\| \\
\beta:=\max _{j>\ell-r}\left\|B_{r, j}^{(\ell)}\right\|
\end{array},\right.
$$

which follows by (32) and the fact that the matrices $A_{r, j}^{(\ell)}$ are nonnegative.

Step 2. For every $r$ and $j$, the $k$ th column in $B_{r, j}^{(\ell)}$ is null when $k>2 r+2 j-2$.

Proof. By induction on $r$. When $r=1$ the claim holds because $B_{1, j}^{(\ell)}=$ $A_{1, j}^{(\ell)}=A_{j}^{(\ell)}$ has a unique non zero row, the $j$ th one, and this row is $\left[\frac{1}{2 j-1}\right]_{\ell}$ whose $k$ th entry is zero if $k>2 j$. Suppose the claim holds for $r$, and that $k>2(r+1)+2 j-2$. By (33) we have:

$$
\begin{aligned}
\left\|B_{r+1, j}^{(\ell)}\right\|_{k} & =\left\|A_{1, j}^{(\ell)}\right\|_{k}+\left\|B_{r, 1}^{(\ell)} A_{1, j}^{(\ell)}\right\|_{k}+\left\|B_{r, j+1}^{(\ell)}\right\|_{k} \\
& =\left\|A_{1, j}^{(\ell)}\right\|_{k}+\frac{1}{(2 j-k) !}\left\|B_{r, 1}^{(\ell)}\right\|_{j}+\left\|B_{r, j+1}^{(\ell)}\right\|_{k} .
\end{aligned}
$$

In this decomposition $\left\|B_{r, j+1}^{(\ell)}\right\|_{k}$ is zero by inductive hypothesis (because $k>$ $2 r+2(j+1)-2)$, and also $\left\|A_{1, j}^{(\ell)}\right\|_{k}$ and $\frac{1}{(2 j-k) !}$ are zero, because $k>2 j$. 
Step 3. From (33) we obtain that $B_{r, j}^{(\ell)} \leq\left(\mathbb{I}_{\ell}+B_{r, 1}^{(\ell)}\right) A_{1, j}^{(\ell)}+B_{r, j+1}^{(\ell)}$, because every $A_{r, j}^{(\ell)}$ is nonnegative. Iterating this inequality we get:

$$
B_{r, j}^{(\ell)} \leq\left(\mathbb{I}_{\ell}+B_{r, 1}^{(\ell)}\right)\left(A_{1, j}^{(\ell)}+\cdots+A_{1, \ell}^{(\ell)}\right) .
$$

By (35) we know that $\left\|\mathbb{I}_{\ell}+B_{r, 1}^{(\ell)}\right\| \leq\left\|S_{\ell}^{r}\right\|$, and $\left\|A_{1, j}^{(\ell)}+\cdots+A_{1, \ell}^{(\ell)}\right\|$ is lower than $\mathrm{Ch}(1)-1$ when $2 j \geq \ell+2$, hence the previous inequality gives:

$$
\left\|B_{r, j}^{(\ell)}\right\| \leq(\mathrm{Ch}(1)-1)\left\|S_{\ell}^{r}\right\|, \quad \text { if } 2 j \geq \ell+2 .
$$

We do not know the exact value of $\left\|S_{\ell}^{r}\right\|$, however $\left\|S_{\ell}^{r}\right\|^{1 / r}$ is always greater than $\lambda_{\ell}$ (the spectral radius of $S_{\ell}$ ) and this sequence grows with $\ell$, hence $\left\|S_{\ell}^{r}\right\|^{1 / r} \geq \lambda_{2}=1.184 \ldots$. Therefore, if $r \geq 6$ we have $\left\|S_{\ell}^{r}\right\| \geq \lambda_{2}^{6}>(2-$ $\mathrm{Ch}(1))^{-1}$ and from (36) we get that:

$$
1+\left\|B_{r, j}^{(\ell)}\right\|<\left\|S_{\ell}^{r}\right\| \quad \text { when } 2 j \geq \ell+2 \text { and } r \geq 6 .
$$

This means that for $\ell \geq 2 r$ and $r \geq 6$ in the formula (35) for the norm of $S_{\ell}^{r}$ only the matrices $B_{r, j}^{(\ell)}$ with $j \leq\lceil\ell / 2\rceil$ matter, and it becomes:

$$
\left\|S_{\ell}^{r}\right\|=1+\max _{j \leq\lceil\ell / 2\rceil}\left\|B_{r, j}^{(\ell)}\right\|=1+\max _{j \leq\lceil\ell / 2\rceil} \max _{u \leq \ell}\left\|B_{r, j}^{(\ell)}\right\|_{u}, \quad \text { when } \ell \geq 2 r, r \geq 6 .
$$

Step 4. We prove that:

$$
B_{r, j}^{(\ell)}=\left(\begin{array}{cc}
B_{r, j}^{(\ell-1)} & * \\
O_{1 \times \ell-1} & 0
\end{array}\right) \quad \text { when } \ell>r+j-1 .
$$

Proof. By induction on $r$. When $r=1$ the claim follows at once by the definition of $A_{j}^{(\ell)}$ and the equality $B_{1, j}^{(\ell)}=A_{j}^{(\ell)}$. Let the claim be true for $r$ and suppose that $\ell>(r+1)+j-1$. Then $\ell>r+(j+1)-1$, therefore the last row of $B_{r, 1}^{(\ell)}, A_{1, j}^{(\ell)}$ and $B_{r, j+1}^{(\ell)}$ is null and the claim for $B_{r+1, j}^{(\ell)}$ follows by (33).

Step 5. From (34) we have for the norm of the $u$ th column in $B_{r, j}^{(\ell)}$ the representation:

$$
\left\|B_{r, j}^{(\ell)}\right\|_{u}=\sum_{\kappa=j}^{r+j-1} \frac{1}{(2 \kappa-u) !}\left(1+\left\|B_{r+j-1-\kappa, 1}^{(\ell)}\right\|_{\kappa}\right) .
$$

In this sum the terms with $\kappa<u / 2$ do not contribute, hence:

$$
\left\|B_{r, j}^{(\ell)}\right\|_{u}=\sum_{\kappa=\max \{j,\lceil u / 2\rceil\}}^{r+j-1} \frac{1}{(2 \kappa-u) !}\left(1+\left\|B_{r+j-1-\kappa, 1}^{(\ell)}\right\|_{\kappa}\right) \quad \forall u, r, \ell .
$$

This formula shows that $\left\|B_{r, j}^{(\ell)}\right\|_{u}$ depends only on $r+j$ when $j \leq\lceil u / 2\rceil$; in particular, $\left\|B_{r, j}^{(\ell)}\right\|_{u}=\left\|B_{r+j-1,1}^{(\ell)}\right\|_{u}$ when $j \leq\lceil u / 2\rceil$.

Step 6. By (38) we can restrict our attention to the matrices $B_{r, j}^{(\ell)}$ with $j \leq\lceil\ell / 2\rceil$. Under this assumption and furthermore assuming that $r \geq 22$ and $\ell \geq 2 r$, we prove now that $1+\left\|B_{r, j}^{(\ell)}\right\|_{\ell}<\left\|S_{\ell}^{r}\right\|$, i.e. that the starred column in (39) does not contribute to the norm of $S_{\ell}^{r}$ and can be neglected. 
Proof. When $j \leq\lceil\ell / 2\rceil$ we have $\left\|B_{r, j}^{(\ell)}\right\|_{\ell}=\left\|B_{r+j-1,1}^{(\ell)}\right\|_{\ell}$, by Step 5. Furthermore, by (40) we see that in order to evaluate $\left\|B_{r+j-1,1}^{(\ell)}\right\|_{\ell}$ we must evaluate $\left\|B_{r+j-1-\kappa, 1}^{(\ell)}\right\|_{\kappa}$ : this suggests an iterative process, here. For every integer $t$, we define the constants $h_{t}$ and $\kappa_{t}$ as:

$$
\begin{aligned}
\kappa_{0} & =\ell, & h_{0} & =r+j-1, \\
\kappa_{t} & \in\left[\left\lceil\kappa_{t-1} / 2\right\rceil, h_{t-1}\right], & h_{t} & =h_{t-1}-\kappa_{t} .
\end{aligned}
$$

Then, by (40) again, we have for every $t \geq 1$ that:

$$
\left\|B_{h_{t-1}, 1}^{(\ell)}\right\|_{\kappa_{t-1}}=\sum_{\kappa_{t}=\left\lceil\kappa_{t-1} / 2\right\rceil}^{h_{t-1}} \frac{1}{\left(2 \kappa_{t}-\kappa_{t-1}\right) !}\left(1+\left\|B_{h_{t}, 1}^{(\ell)}\right\|_{\kappa_{t}}\right) .
$$

Suppose that $\left(2^{u+1}-1\right) \kappa_{t-u}>2^{u+1} h_{t-u}$ holds for some integer $u \in[0, t-1]$, then $\kappa_{t}>2 h_{t}$. In fact, this is evident if $u=0$, and

$$
\left(2^{u+2}-1\right) \kappa_{t-u-1}>2^{u+2} h_{t-u-1} \Longleftrightarrow\left(2^{u+1}-1\right) \frac{\kappa_{t-u-1}}{2}>2^{u+1}\left(h_{t-u-1}-\frac{\kappa_{t-u-1}}{2}\right)
$$

which implies that $\left(2^{u+1}-1\right) \kappa_{t-u}>2^{u+1} h_{t-u}$; the claim follows now by a descent argument on $u$. In particular, if $\left(2^{t}-1\right) \kappa_{1}>2^{t} h_{1}$, then $\kappa_{t}>2 h_{t}$ (take $u=t-1$ in the previous argument). Since $\kappa_{1} \geq\lceil\ell / 2\rceil$ and $h_{1} \leq r-1$, we obtain that $\kappa_{t}>2 h_{t}$ when $\ell>\frac{2^{t+1}}{2^{t}-1}\left(r-1\right.$ ), so that $\left\|B_{h_{t}, 1}^{(\ell)}\right\|_{\kappa_{t}}=0$ (by Step 2), under that hypothesis. By induction on $t$, using (41), we deduce that:

$$
\text { if } \quad \ell>\frac{2^{t+1}}{2^{t}-1}(r-1) \text { then }\left\|B_{h_{0}, 1}^{(\ell)}\right\|_{\kappa_{0}} \leq \gamma_{t},
$$

where $\gamma_{t}$ is the sequence defined as:

$$
\gamma_{0}=0, \quad \gamma_{s+1}=\left(1+\gamma_{s}\right) \operatorname{Ch}(1) \quad \forall s \geq 0 .
$$

Hence we have proved that:

if $\quad \ell>\frac{2^{t+1}}{2^{t}-1}(r-1)$ then $\left\|B_{r, j}^{(\ell)}\right\|_{\ell}=\left\|B_{h_{0}, 1}^{(\ell)}\right\|_{\kappa_{0}} \leq \frac{\mathrm{Ch}(1)}{\operatorname{Ch}(1)-1}(\mathrm{Ch}(1))^{t}$,

because $\gamma_{s} \leq \frac{\mathrm{Ch}(1)}{\operatorname{Ch}(1)-1}(\mathrm{Ch}(1))^{s}$ for every integer $s$. Moreover, $\left\|S_{\ell}^{r}\right\| \geq \lambda_{2}^{r}$ and $\lambda_{2}=1.184 \ldots$, therefore if $r>t \frac{\log \mathrm{Ch}(1)}{\log \lambda_{2}}+10$ then

$$
1+\left\|B_{r, j}^{(\ell)}\right\|_{\ell} \leq 1+\frac{\mathrm{Ch}(1)}{\operatorname{Ch}(1)-1}(\mathrm{Ch}(1))^{t}<\lambda_{2}^{r} \leq\left\|S_{\ell}^{r}\right\| .
$$

We have obtained this inequality under the assumptions $\ell>\frac{2^{t+1}}{2^{t}-1}(r-1)$ and $r>t \frac{\log \mathrm{Ch}(1)}{\log \lambda_{2}}+10$, and these inequalities hold for some $t$ when $\ell \geq 2 r$ and $r \geq 22$.

Step 7. Let $\ell \geq 2 r+1$ and $r \geq 22$. Then by (38) we have both

$$
\left\|S_{\ell-1}^{r}\right\|-1=\max _{j \leq\lceil(\ell-1) / 2\rceil} \max _{u \leq \ell-1}\left\|B_{r, j}^{(\ell-1)}\right\|_{u},
$$

and

$$
\left\|S_{\ell}^{r}\right\|-1=\max _{j \leq\lceil\ell / 2\rceil} \max _{u \leq \ell}\left\|B_{r, j}^{(\ell)}\right\|_{u}
$$


In (42) we can extend the range of $j$ to $j \leq\lceil\ell / 2\rceil$ : this is evident when $\ell$ is even, thus suppose $\ell=2 q+1$ for some integer $q \geq r$. The extension to $j \leq\lceil\ell / 2\rceil$ adds a unique term to the range: that one with $j=q+1$, but $\left\|B_{r, q+1}^{(\ell-1)}\right\|<\left\|S_{\ell-1}^{r}\right\|-1$ by $(37)$, because $2(q+1) \geq(\ell-1)+2$.

Moreover, by Step 6 and under the present assumptions we have $\left\|B_{r, j}^{(\ell)}\right\|_{\ell}<$ $\left\|S_{\ell}^{r}\right\|-1$, therefore in (43) we can restrict $u$ to $u \leq \ell-1$. Furthermore, by (39) we have also that $\left\|B_{r, j}^{(\ell)}\right\|_{u}=\left\|B_{r, j}^{(\ell-1)}\right\|_{u}$ when $u \leq \ell-1$ : these remarks prove that RHS in (42) and (43) are both equal to $\max _{j \leq\lceil\ell / 2\rceil} \max _{u \leq \ell-1}\left\|B_{r, j}^{(\ell-1)}\right\|_{u}$, so that the equality $\left\|S_{\ell-1}^{r}\right\|=\left\|S_{\ell}^{r}\right\|$ follows.

Step 8. Let $r \geq 22$; the previous steps and (38) prove that:

$$
\Lambda_{r, \infty}^{r}=\left\|S_{2 r}^{r}\right\|=1+\max _{j \leq r} \max _{u \leq 2 r}\left\|B_{r, j}^{(2 r)}\right\|_{u} .
$$

We prove now that in this formula we can restrict $u$ to the unique value $2 j$, i.e. that:

$$
\Lambda_{r, \infty}^{r}=\left\|S_{2 r}^{r}\right\|=1+\max _{j \leq r}\left\|B_{r, j}^{(2 r)}\right\|_{2 j} .
$$

In fact, when $u \leq 2 j$, by (40) we have:

$$
\begin{aligned}
\left\|B_{r, j}^{(2 r)}\right\|_{u} & =\sum_{\kappa=j}^{r+j-1} \frac{1}{(2 \kappa-u) !}\left(1+\left\|B_{r+j-1-\kappa, 1}^{(2 r)}\right\|_{\kappa}\right) \\
& \leq \sum_{\kappa=j}^{r+j-1} \frac{1}{(2 \kappa-2 j) !}\left(1+\left\|B_{r+j-1-\kappa, 1}^{(2 r)}\right\|_{\kappa}\right)=\left\|B_{r, j}^{(2 r)}\right\|_{2 j},
\end{aligned}
$$

so that the columns of index below $2 j$ are dominated by the $2 j$ th one. Moreover, the recursive law for $A_{r, j}^{(2 r)}$ gives $\left\|A_{r, j}^{(2 r)}\right\|_{u} \geq\left\|A_{r-1, j+1}^{(2 r)}\right\|_{u}$ for every $r, j$ and $u$. Iterating this inequality we get $\left\|A_{r, j}^{(2 r)}\right\|_{u} \geq\left\|A_{1, r+j-1}^{(2 r)}\right\|_{u}=$ $\frac{1}{(2 r+2 j-2-u) !}$ proving that $\left\|A_{r, j}^{(2 r)}\right\|_{u}$ is always strictly positive. This implies that $\left\|S_{2 r}^{r-1}\right\|$ is strictly lower than $\left\|S_{2 r}^{r}\right\|$. In fact,

$$
\begin{aligned}
\left\|S_{2 r}^{r-1}\right\| & =\left\|S_{2 r-1}^{r-1}\right\| & & \text { by }(44), \\
& =1+\max _{j \leq r}\left\|B_{r-1, j}^{(2 r-1)}\right\| & & \text { by }(38), \\
& \leq 1+\max _{j \leq r}\left\|B_{r-1, j}^{(2 r)}\right\| & & \text { by }(39), \\
& <1+\max _{j \leq r}\left\|B_{r, j}^{(2 r)}\right\| & & \text { because }\left\|A_{r, j}^{(2 r)}\right\|_{u}>0 \text { for every } r, j \text { and } u, \\
& =\left\|S_{2 r}^{r}\right\| & & \text { by }(38) .
\end{aligned}
$$

Suppose now $u>2 j$ (and then $j<r$ ). Then $\left\|B_{r, j}^{(2 r)}\right\|_{u}=\left\|B_{r-1, j+1}^{(2 r)}\right\|_{u}$ by (40), and by (38):

$$
\left\|S_{2 r}^{r-1}\right\|=1+\max _{j \leq r} \max _{u \leq 2 r}\left\|B_{r-1, j}^{(2 r)}\right\|_{u}
$$

so that $\left\|B_{r-1, j+1}^{(2 r)}\right\|_{u} \leq\left\|S_{2 r}^{r-1}\right\|-1$ when $j<r$. Therefore, under those assumptions we get:

$$
1+\left\|B_{r, j}^{(2 r)}\right\|_{u}=1+\left\|B_{r-1, j+1}^{(2 r)}\right\|_{u} \leq\left\|S_{2 r}^{r-1}\right\|<\left\|S_{2 r}^{r}\right\|, \quad \text { when } u>2 j .
$$


This shows that in (44) only the columns $u \leq 2 j$ matter, so that (45) is proved.

Introducing in (45) the formula (40) for $\left\|B_{r, j}^{(2 r)}\right\|_{2 j}$ we get:

$$
\Lambda_{r, \infty}^{r}=\left\|S_{2 r}^{r}\right\|=1+\max _{j \leq r}\left\{\sum_{\kappa=j}^{r+j-1} \frac{1}{(2 \kappa-2 j) !}\left(1+\left\|B_{r+j-1-\kappa, 1}^{(2 r)}\right\|_{\kappa}\right)\right\},
$$

which becomes (30) by setting $d_{i, u}:=\left\|B_{i, 1}^{(2 r)}\right\|_{u}$ : the recursive law (29) for $d_{i, u}$ is an immediate consequence of (40). At last, the bound in (31) follows by (30) and the fact that $d_{r-1-\kappa, j+\kappa} \leq d_{r-1, j+\kappa}$ for every $r, j$ and $\kappa$.

The previous proposition reduces the search of an upper bound for $\Lambda_{\infty}$ to a search of an upper bound for $d_{i, u}$. The recursive definition (29) suggests the need to look for a bound of the form:

$$
d_{i, u} \leq \alpha \beta^{i} \quad \forall i, u .
$$

In fact, it can be proved with $\alpha=50$ and $\beta=1.8$. However, the values of $d_{i, u}$ for fixed $i$ and $u$ varying in $1, \ldots, 2 i$ manifest a very complex behavior that is not captured by any bound of the form (46). For example, the following picture shows that the quotients $d_{i, u+1} / d_{i, u}$ have a complicated and band-like structure:

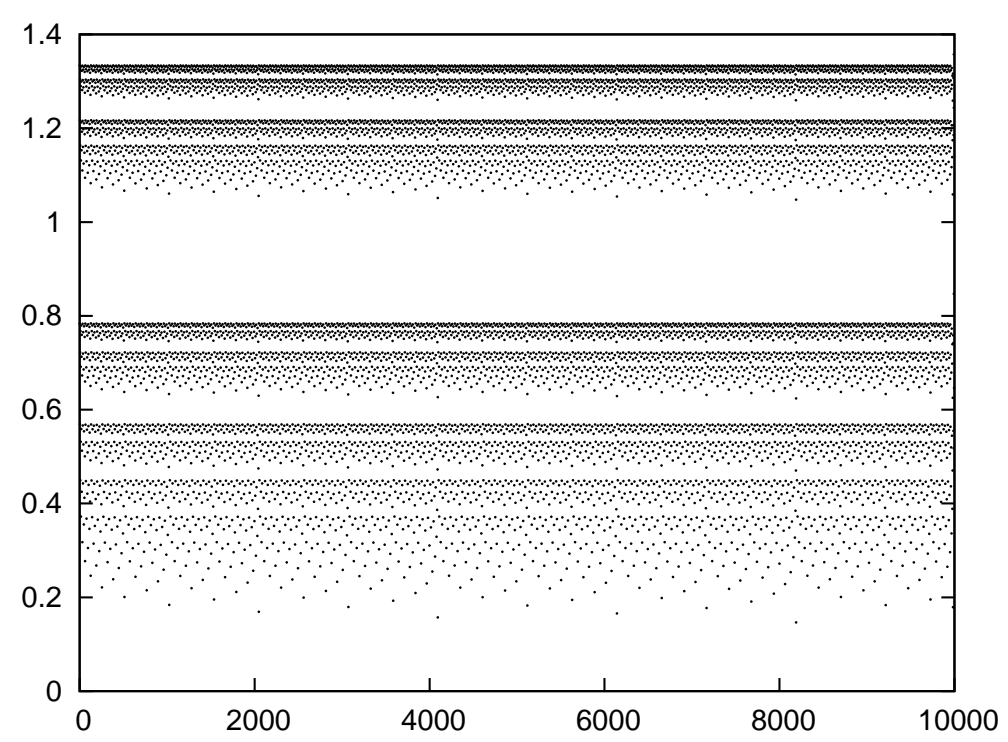

Figure: values of $d_{10000, u+1} / d_{10000, u}$ for $u \leq 10000$.

Moreover, similar pictures with different values of $i$ show that this structure is essentially independent of the value of $i$ when it is large enough. These facts suggest the existence of an upper bound similar to (46) but with a coefficient $\alpha$ which is a periodic function of $u$, i.e., the existence of an integer $\ell$, of an $\ell$-periodic function $\bar{\alpha}: \mathbb{N} \rightarrow \mathbb{R}^{+}$, and of a constant $\beta_{\ell}$ such that:

$$
d_{i, u} \leq \bar{\alpha}_{\ell}(u) \beta_{\ell}^{i} \quad \forall i, u \text {. }
$$


If this bound is confirmed, then due to the boundedness of the coefficients $\bar{\alpha}_{\ell}(u)$ and via (31), we can conclude that:

$$
\Lambda_{\infty} \leq \beta_{\ell}
$$

Suppose that for certain $\ell, \bar{\alpha}_{\ell}$ and $\beta$ the upper bound in (47) holds up to $i$ - 1 , with $i>\ell$. Then, the recursive definition of $d_{i, u}$ gives:

$$
\begin{aligned}
d_{i, u} & \leq \operatorname{Ch}(1)+\sum_{\kappa=\lceil u / 2\rceil}^{i} \frac{d_{i-\kappa, \kappa}}{(2 \kappa-u) !} \\
& \leq \operatorname{Ch}(1)+\sum_{\kappa=\lceil u / 2\rceil}^{i} \bar{\alpha}_{\ell}(\kappa) \frac{\beta^{i-\kappa}}{(2 \kappa-u) !} \\
& \leq \operatorname{Ch}(1)+\sum_{j=1}^{\ell} \bar{\alpha}_{\ell}(j) \sum_{\substack{\kappa \geq\lceil u / 2\rceil \\
\kappa=j(\bmod \ell)}}^{+\infty} \frac{\beta^{i-\kappa}}{(2 \kappa-u) !} \\
& \leq \operatorname{Ch}(1)+\beta^{i-u / 2} \sum_{j=1}^{\ell} \bar{\alpha}_{\ell}(j) F_{u, j}\left(\beta^{-1 / 2}\right)
\end{aligned}
$$

where

$$
F_{u, j}(x):=\sum_{\substack{\kappa \geq\lceil u / 2\rceil \\ \kappa=j(\bmod \ell)}}^{+\infty} \frac{x^{2 \kappa-u}}{(2 \kappa-u) !}=\sum_{\substack{\kappa \geq \max \{0,2 j-u\} \\ \kappa=2 j-u(\bmod 2 \ell)}}^{+\infty} \frac{x^{\kappa}}{\kappa !}=: G_{2 j-u}^{(2 \ell)}(x) .
$$

Hence, the inequality $d_{i, u} \leq \bar{\alpha}_{\ell}(u) \beta^{i}$ holds whenever

$$
\frac{\mathrm{Ch}(1)}{\beta^{i}}+\beta^{-u / 2} \sum_{j=1}^{\ell} \bar{\alpha}_{\ell}(j) F_{u, j}\left(\beta^{-1 / 2}\right) \leq \bar{\alpha}_{\ell}(u) \quad \forall u \leq 2 i .
$$

The constant $\beta$ is certainly larger then $1, \bar{\alpha}_{\ell}$ is $\ell$-periodic by hypothesis and $F_{u, j}$ is $2 \ell$-periodic in $u$, therefore the inequality will follow at once if

$$
\frac{\mathrm{Ch}(1)}{\beta}+\beta^{-u / 2} \sum_{j=1}^{\ell} \bar{\alpha}_{\ell}(j) F_{u, j}\left(\beta^{-1 / 2}\right) \leq \bar{\alpha}_{\ell}(u) \quad u=1, \ldots, 2 \ell .
$$

Suppose that $\ell, \alpha_{\ell}: \mathbb{N} \rightarrow \mathbb{C}$ and $\beta$ satisfy:

$$
\beta^{-u / 2} \sum_{j=1}^{\ell} \alpha_{\ell}(j) F_{u, j}\left(\beta^{-1 / 2}\right)<\alpha_{\ell}(u) \quad u=1, \ldots, 2 \ell,
$$

then it is always possible to set a positive constant $\gamma$ such that (49) holds with $\bar{\alpha}_{\ell}=\gamma \alpha_{\ell}$; furthermore, $\gamma$ can also be chosen large enough so that the inequality $d_{i, u} \leq \bar{\alpha}_{\ell}(u) \beta^{i}$ holds for every $i \leq \ell$ and $u \leq 2 i$. Thus, if we are able to find $\ell, \alpha_{\ell}$ and $\beta$ such that (50) holds, then we have immediately an inequality of the form (47) and the upper bound (48). This means that our goal now is to find $\ell$ and $\alpha_{\ell}$ such that (50) holds with $\beta$ as small as possible.

The inequalities in (50) can be written in a more compact way. Let $\mathcal{F}_{\ell}$ and $\overline{\mathcal{F}}_{\ell}$ be the square matrices of order $\ell$ defined by:

$$
\mathcal{F}_{\ell}(x):=\left(x^{u} F_{u, j}(x)\right)_{u=1, j=1}^{\ell, \ell}, \quad \overline{\mathcal{F}}_{\ell}(x):=\left(x^{u} F_{u, j}(x)\right)_{u=\ell+1, j=1}^{2 \ell, \ell} .
$$


Then (50) reads

$$
\mathcal{F}_{\ell}(x) \boldsymbol{\alpha}^{(\ell)}<\boldsymbol{\alpha}^{(\ell)}, \text { and } \overline{\mathcal{F}}_{\ell}(x) \boldsymbol{\alpha}^{(\ell)}<\boldsymbol{\alpha}^{(\ell)},
$$

where $\boldsymbol{\alpha}^{(\ell)}$ denotes the vector $\left(\alpha_{\ell}(1), \ldots, \alpha_{\ell}(\ell)\right)^{\top}$, and $x=1 / \sqrt{\beta}$. This means that, for a given $\ell$, we are looking for an $x$ such that (51) holds true for some vector $\boldsymbol{\alpha}^{(\ell)}$ with positive entries. The next result shows that we can satisfy the first inequality in (51) with an $x$ arbitrarily close to $1 / \sqrt{\lambda_{\infty}}$ if $\ell$ is large enough; the next main difficulty will be proving that, under some circumstances, the same values also satisfy the second inequality.

Lemma 7. There exists a unique $x_{\ell} \in(0,1)$ such that $\mathcal{F}_{\ell}\left(x_{\ell}\right)$ has 1 as eigenvalue and a positive vector $\boldsymbol{\alpha}^{(\ell)}$ as eigenvector. Moreover,

$$
\left|x_{\ell}-1 / \sqrt{\lambda_{\infty}}\right| \leq \frac{1.1}{0.677}\left(\frac{x_{\ell}^{2} e}{\sqrt{\ell}}\right)^{\ell} \quad \forall \ell \geq 16,
$$

so that $x_{\ell}$ tends to $1 / \sqrt{\lambda_{\infty}}$ as $\ell$ diverges and $\left|x_{\ell}-1 / \sqrt{\lambda_{\infty}}\right| \leq 10^{-3}$ for every $\ell \geq 16$.

It is convenient to introduce $\bar{x}$ to denote the constant 0.917 , so that Lemma 7 and the explicit evaluation in Section 4 imply $x_{\ell} \leq \bar{x}$ for every $\ell \geq 16$. Moreover, here and later in Lemma 10, we adopt the notation $f(x)=\bar{O}(g(x))$ as equivalent to $|f(x)| \leq g(x)$.

Proof. The matrix $\mathcal{F}_{\ell}(x)$ coincides essentially with the transpose of the matrix $\mathcal{A}_{\ell}\left(x^{2}\right)$, which is defined in (12). In fact, the $u, j$ entry in $\mathcal{A}_{\ell}^{\top}\left(x^{2}\right)$ coincides with the first term of the power series representing $x^{u} G_{2 j-u}^{(2 \ell)}(x)$ when $2 j-u \geq 0$ and is 0 otherwise so that $\mathcal{F}_{\ell}(x)-\mathcal{A}_{\ell}^{\top}\left(x^{2}\right)=\mathcal{B}_{\ell}(x)$ with:

We notice that:

$$
\mathcal{B}_{\ell}(x):=\left\{x^{u} G_{2 \ell+2 j-u}^{(2 \ell)}(x)\right\}_{u=1, j=1}^{\ell, \ell} .
$$

$$
x^{u} G_{2 \ell+2 j-u}^{(2 \ell)}(x)=\sum_{t=0}^{+\infty} \frac{x^{2 \ell+2 j+2 \ell t}}{(2 \ell+2 j-u+2 \ell t) !} \leq \frac{x^{2 \ell+2 j}}{(2 \ell+2 j-u) !} G_{0}^{(2 \ell)}(x),
$$

and that:

$$
G_{0}^{(2 \ell)}(x)=\sum_{t=0}^{+\infty} \frac{x^{2 \ell t}}{(2 \ell t) !} \leq 1+\frac{1}{(2 \ell) !} \sum_{t=1}^{+\infty} \frac{x^{2 \ell t}}{t !}=1+\frac{e^{x^{2 \ell}}-1}{(2 \ell) !} \leq 2
$$

for $x \in[0,1]$ and $\ell \geq 1$, so that each entry in $\mathcal{B}_{\ell}(x)$ is lower than $2 x^{2 \ell} /(\ell+2)$ !.

Now we compute $\operatorname{det}\left(\mathbb{I}_{\ell}-\mathcal{F}_{\ell}(x)\right)$, i.e. $\operatorname{det}\left(\mathbb{I}_{\ell}-\mathcal{A}_{\ell}^{\top}\left(x^{2}\right)-\mathcal{B}_{\ell}(x)\right)$, using the Laplace formula with respect to the last column. When, in this formula, we take only elements in $\mathcal{A}_{\ell}^{\top}\left(x^{2}\right)$, we reproduce $\operatorname{det}\left(\mathbb{I}_{\ell}-\mathcal{A}_{\ell}^{\top}\left(x^{2}\right)\right)$, which is $q_{\ell}\left(x^{2}\right)$. Therefore, the difference $\operatorname{det}\left(\mathbb{I}-\mathcal{F}_{\ell}(x)\right)-q_{\ell}\left(x^{2}\right)$ is due to the terms coming from $\mathcal{B}_{\ell}(x)$. These terms contribute in two different ways. The first contribution is of the form:

$$
\sum_{u=1}^{\ell}\left[\mathcal{B}_{\ell}(x)\right]_{u, \ell} \cdot\{u, \ell \text {-cofactor }\}
$$

The second one is of the form:

$$
\sum_{u=1}^{\ell}\left[\mathbb{I}_{\ell}-\mathcal{A}_{\ell}^{\top}\left(x^{2}\right)\right]_{u, \ell} \cdot\left\{\begin{array}{l}
\text { terms of the } u, \ell \text {-cofactor containing } \\
\text { at least one factor from } \mathcal{B}_{\ell}(x)
\end{array}\right\} .
$$


The absolute value of every $u, j$ entry in $\mathbb{I}_{\ell}-\mathcal{F}_{\ell}(x)$ is lower than 2 when $u \in$ $\{2 j, 2 j-1\}$ and than 1 in each other case; hence, using the Hadamard inequality (see [1], Th. II.3.17), the cofactor in the first contribution can be bounded as $\left(2^{2}+\right.$ $\left.2^{2}+\ell-3\right)^{(\ell-1) / 2}$, while the terms of the second contribution are bounded by $\ell^{2}\left(2^{2}+\right.$ $\left.2^{2}+\ell-4\right)^{(\ell-2) / 2} 2 x^{2 \ell} /(\ell+2)$ ! (because there are $(\ell-1)^{2}$ possible choices for the position where the $\mathcal{B}_{\ell}(x)$ is taken, every such term is bounded using Hadamard, and each entry in $\mathcal{B}_{\ell}(x)$ is lower than $2 x^{2 \ell} /(\ell+2)$ !). As a consequence,

$$
\begin{aligned}
& \left|\operatorname{det}\left(\mathbb{I}_{\ell}-\mathcal{F}_{\ell}(x)\right)-q_{\ell}\left(x^{2}\right)\right| \\
& \leq \sum_{u=1}^{\ell}\left[\mathcal{B}_{\ell}(x)\right]_{u, \ell} \cdot(\ell+5)^{(\ell-1) / 2}+\sum_{u=1}^{\ell}\left|\left[\mathbb{I}_{\ell}-\mathcal{A}_{\ell}^{\top}\left(x^{2}\right)\right]_{u, \ell}\right| \cdot \frac{2 x^{2 \ell}}{(\ell+2) !} \cdot \ell^{2}(\ell+4)^{(\ell-2) / 2} .
\end{aligned}
$$

Using (53) and recalling that $\ell ! \geq e(\ell / e)^{\ell}$, we get:

$$
\begin{aligned}
& \leq \sum_{u=1}^{\ell} \frac{2 x^{4 \ell}}{(4 \ell-u) !} \cdot(\ell+5)^{\frac{\ell-1}{2}}+\left[\sum_{u=1}^{\ell-1} \frac{x^{2 \ell}}{(2 \ell-u) !}+\left(1-\frac{x^{2 \ell}}{\ell !}\right)\right] \cdot \frac{2 x^{2 \ell} \ell^{2}(\ell+4)^{\frac{\ell-2}{2}}}{(\ell+2) !} \\
& \leq \frac{2 e^{5 / 2} x^{4 \ell}}{\sqrt{\ell+5}}\left(\frac{(e / 3)^{3}}{\ell^{5 / 2}}\right)^{\ell}+\frac{2 x^{4 \ell}}{(\ell+1)^{2}}\left(\frac{e^{2}}{\ell^{3 / 2}}\right)^{\ell}+\frac{2 e x^{2 \ell}}{\ell+4}\left(\frac{e}{\sqrt{\ell}}\right)^{\ell} .
\end{aligned}
$$

This bound proves that:

$$
\operatorname{det}\left(\mathbb{I}_{\ell}-\mathcal{F}_{\ell}(x)\right)=q_{\ell}\left(x^{2}\right)+\bar{O}\left(\left(\frac{x^{2} e}{\sqrt{\ell}}\right)^{\ell}\right) \quad \forall x \in[0,1]
$$

when $\ell \geq 16$.

This identity shows that $\operatorname{det}\left(\mathbb{I}_{\ell}-\mathcal{F}_{\ell}(1)\right)$ is negative when $\ell \geq 16$ because $q_{\ell}(1)<$ -0.08 for every $\ell \geq 6$ (based on Lemma 3, we know that $\left|q_{\ell}(1)-q_{6}(1)\right|<0.05$ when $\ell \geq 6$ and a computation shows that $\left.q_{6}(1)<-0.13\right)$, and $\left(\frac{e}{\sqrt{\ell}}\right)^{\ell}<0.01$. Therefore, the equation:

$$
\operatorname{det}\left(\lambda \mathbb{I}_{\ell}-\mathcal{F}_{\ell}(1)\right)=0
$$

admits a real solution $\lambda$ greater than 1 and the spectral radius of $\mathcal{F}_{\ell}(1)$ itself is strictly larger than 1. Since the spectral radius of $\mathcal{F}_{\ell}(x)$ is a continuous and monotone function of $x$ (as a consequence of the Perron-Frobenius theorem) and since $\mathcal{F}_{\ell}(0)$ is the null matrix, we conclude that there exists a unique $x_{\ell} \in(0,1)$ for which the spectral radius of $\mathcal{F}_{\ell}\left(x_{\ell}\right)$ is equal to 1 , at least when $\ell \geq 16$. According to the Perron-Frobenius theorem, 1 is an eigenvalue for $\mathcal{F}_{\ell}\left(x_{\ell}\right)$, and its eigenspace is spanned by a positive vector $\boldsymbol{\alpha}^{(\ell)}$. Finally, the relations:

$$
0=\operatorname{det}\left(\mathbb{I}_{\ell}-\mathcal{F}_{\ell}\left(x_{\ell}\right)\right)=q_{\ell}\left(x_{\ell}^{2}\right)+\bar{O}\left(\left(\frac{x_{\ell}^{2} e}{\sqrt{\ell}}\right)^{\ell}\right) \quad \text { and } \quad 0=q_{\ell}\left(\lambda_{\ell}^{-1}\right)
$$

give:

$$
\left(\lambda_{\ell}^{-1}-x_{\ell}^{2}\right) q_{\ell}^{\prime}(\zeta)=q_{\ell}\left(\lambda_{\ell}^{-1}\right)-q_{\ell}\left(x_{\ell}^{2}\right)=\bar{O}\left(\left(\frac{x_{\ell}^{2} e}{\sqrt{\ell}}\right)^{\ell}\right),
$$

for a suitable $\zeta \in(0,1)$. The derivative of $q_{\ell}(x)$ is greater than 0.677 uniformly in $x \in(0,1)$ and $\ell$ (see Section 4), and $\left|\lambda_{\ell}^{-1}-\lambda_{\infty}^{-1}\right| \leq \frac{1.51}{0.677}\left(\frac{e}{\ell}\right)^{\ell}$ as $\ell \geq 16$ (by (24)). With the previous inequality, we get:

$$
\left|x_{\ell}-\sqrt{1 / \lambda_{\infty}}\right| \leq \frac{\sqrt{\lambda_{\infty}}}{0.677}\left[\left(\frac{x_{\ell}^{2} e}{\sqrt{\ell}}\right)^{\ell}+1.51\left(\frac{e}{\ell}\right)^{\ell}\right] .
$$


For $\ell \geq 16$, this inequality shows that $x_{\ell} \geq \sqrt{1 / \lambda_{\infty}}-10^{-3}$. We get (52) by substituting this lower bound in the previous inequality and using the value of $\lambda_{\infty}$ we computed in Section 4.

Given the result in the previous lemma, our strategy is now evident: proving that the eigenvector $\boldsymbol{\alpha}^{(\ell)}$ also satisfies the second requirement $\overline{\mathcal{F}}_{\ell}\left(x_{\ell}^{\prime}\right) \boldsymbol{\alpha}^{(\ell)}<\boldsymbol{\alpha}^{(\ell)}$ for a suitable $x_{\ell}^{\prime} \leq x_{\ell}$ with $x_{\ell}^{\prime}-x_{\ell}=o(1)$, at least for infinitely many $\ell$. We will reach this goal with a careful analysis of the matrix $\mathcal{F}_{\ell}$.

From now on, we assume that $\ell$ is even. We write

$$
\mathcal{F}_{\ell}(x) \quad \text { as } \quad\left(\begin{array}{cc}
\mathcal{H}_{\ell}(x) & \mathcal{K}_{\ell}(x) \\
\mathcal{I}_{\ell}(x) & \mathcal{L}_{\ell}(x)
\end{array}\right)
$$

where each submatrix is a square matrix of order $\ell / 2$. For $x \in[0, \bar{x}]$ we have:

$$
\left\|\mathcal{F}_{\ell}\right\|,\left\|\mathcal{H}_{\ell}\right\|,\left\|\mathcal{H}_{\ell}^{\top}\right\| \leq e, \quad\left\|\mathcal{K}_{\ell}\right\| \leq \frac{x^{\ell / 2}}{(\ell / 2+1) !}, \quad\left\|\mathcal{I}_{\ell}\right\| \leq e x^{\ell / 2}, \quad\left\|\mathcal{L}_{\ell}\right\| \leq x^{\ell}
$$

Moreover, as a consequence of the identity $G_{a}^{(2 \ell)}(x)=G_{a}^{(4 \ell)}(x)+G_{a+2 \ell}^{(4 \ell)}(x)$ we get the important relations:

$$
\mathcal{F}_{\ell}=\mathcal{H}_{2 \ell}+\mathcal{K}_{2 \ell}, \quad \overline{\mathcal{F}}_{\ell}=\mathcal{I}_{2 \ell}+\mathcal{L}_{2 \ell}
$$

which suggest that it will be possible to study $\overline{\mathcal{F}}_{\ell}$ via a careful study of $\mathcal{F}_{2 \ell}$. This is the main motivation of the next lemma.

Let $J_{\ell}$ be the square matrix of order $\ell$ whose entries are equal to 1 and recall that:

$$
A J_{\ell} \leq\left\|A^{\top}\right\| J_{\ell}, \quad J_{\ell} A \leq\|A\| J_{\ell}, \quad J_{\ell} J_{\ell}=\ell J_{\ell},
$$

for every square matrix $A$ of order $\ell$.

Lemma 8. For every $\ell \geq 64$, for every $k \geq 0$ and for every $x \in[0, \bar{x}]$, we have:

$$
\mathcal{F}_{2 \ell}^{2^{k}}(x)=\left(\begin{array}{cc}
\mathcal{H}_{2 \ell}^{2^{k}}(x)+O\left(\frac{(2 e)^{2^{k}} x^{\ell}}{(\ell+1) !}\right) J_{\ell} & O\left(\frac{(2 e)^{2^{k}} x^{\ell}}{(\ell+1) !}\right) J_{\ell} \\
O\left((2 e)^{2^{k}} x^{\ell}\right) J_{\ell} & O\left((2 e)^{2^{k}} x^{2 \ell}\right) J_{\ell}
\end{array}\right),
$$

and

$$
\mathcal{F}_{\ell}^{2^{k}}(x)=\mathcal{H}_{2 \ell}^{2^{k}}(x)+O\left(\frac{(2 e)^{2^{k}} x^{\ell}}{(\ell+1) !}\right) J_{\ell}
$$

Proof. By induction on $k$. Let $a_{k}, b_{k}, c_{k}$ and $d_{k}$ recursively defined by $a_{0}=0, b_{0}=1$, $c_{0}=e, d_{0}=1$ and

$$
\left\{\begin{array}{l}
a_{k+1}=2 e^{2^{k}} a_{k}+a_{k}^{2} \frac{x^{\ell}}{\ell !}+b_{k} c_{k} x^{\ell} \ell \\
b_{k+1}=b_{k}\left(e^{2^{k}}+a_{k} \frac{x^{\ell}}{\ell !}+d_{k} x^{2 \ell} \ell\right) \\
c_{k+1}=c_{k}\left(e^{2^{k}}+a_{k} \frac{x^{\ell}}{\ell !}+d_{k} x^{2 \ell} \ell\right) \\
d_{k+1}=b_{k} c_{k} \frac{1}{\ell !}+d_{k}^{2} x^{2 \ell} \ell
\end{array} \quad \forall k \geq 0\right.
$$

Then the equality:

$$
\mathcal{F}_{2 \ell}^{2^{k}}=\left(\begin{array}{cc}
\mathcal{H}_{2 \ell}^{2^{k}}+\bar{O}\left(a_{k} \frac{x^{\ell}}{(\ell+1) !}\right) J_{\ell} & \bar{O}\left(b_{k} \frac{x^{\ell}}{(\ell+1) !}\right) J_{\ell} \\
\bar{O}\left(c_{k} x^{\ell}\right) J_{\ell} & \bar{O}\left(d_{k} x^{2 \ell}\right) J_{\ell}
\end{array}\right)
$$

is proved for every $k$, as a consequence of the equality $\mathcal{F}_{2 \ell}^{2^{k+1}}=\left(\mathcal{F}_{2 \ell}^{2^{k}}\right)^{2}$. The inequalities $a_{k} \leq(2 e)^{2^{k}-1}, b_{k} \leq(2 e)^{2^{k}-1}, c_{k} \leq \frac{1}{2}(2 e)^{2^{k}}$ and $d_{k} \leq(2 e)^{2^{k}-1}$ hold for $k=0$ 
and $k=1$ (here the restriction $x \in[0, \bar{x}]$ is used). By induction, let the inequalities be true for a $k \geq 1$. Then, the inequalities will be true also for $k+1$ if:

$$
\begin{cases}2 \cdot 2^{-2^{k}}+\frac{x^{\ell}}{2 e \ell !}+\frac{1}{2} x^{\ell} \ell & \leq 1 \\ 2^{-2^{k}}+\frac{x^{\ell}}{2 e \ell !}+\frac{1}{2 e} x^{2 \ell} \ell & \leq 1 \\ \frac{1}{2 \ell !}+\frac{1}{2 e} x^{2 \ell} \ell & \leq 1 .\end{cases}
$$

Since we are assuming $k \geq 1$ and $x \leq \bar{x}$, it is immediate to see that these conditions are true for every $\ell \geq 64$. This concludes the proof of the first claim; the second one is proved in similar way, using the identity $\mathcal{F}_{\ell}(x)=\mathcal{H}_{2 \ell}(x)+\mathcal{K}_{2 \ell}(x)=\mathcal{H}_{2 \ell}(x)+$ $\bar{O}\left(\frac{x^{\ell}}{(\ell+1) !}\right) J_{\ell}$.

The previous lemma shows that $\mathcal{F}_{\ell}^{2^{k}}$ and the first $\ell$ components of $\mathcal{F}_{2 \ell}^{2^{k}}$ are very similar. Let $\mathbf{1}_{\ell}$ denote the vector $(1,1, \ldots, 1)^{\top}$ with $\ell$ entries, and given a vector $\boldsymbol{v}$ with an even number of entries, let $\boldsymbol{v}^{+}$and $\boldsymbol{v}^{-}$be the first (second, resp.) half part of $\boldsymbol{v}$, so that $\boldsymbol{v}=:\left(\begin{array}{l}\boldsymbol{v}^{+} \\ \boldsymbol{v}^{-}\end{array}\right)$.

Lemma 9. For every $\ell \geq 64$ and for every $k \geq 0$,

$$
\left[\mathcal{F}_{2 \ell}^{2^{k}}\left(x_{2 \ell}\right) \mathbf{1}_{2 \ell}\right]^{+}-\mathcal{F}_{\ell}^{2^{k}}\left(x_{\ell}\right) \mathbf{1}_{\ell}=O\left(\ell^{2}(2 e)^{2^{k}}\left(\frac{\bar{x}^{2} e}{\sqrt{\ell}}\right)^{\ell}\right) \mathbf{1}_{\ell}
$$

Proof. By Lemmas 7 and 8 we know that:

$$
\left[\mathcal{F}_{2 \ell}^{2^{k}}\left(x_{2 \ell}\right) \mathbf{1}_{2 \ell}\right]^{+}-\mathcal{F}_{\ell}^{2^{k}}\left(x_{\ell}\right) \mathbf{1}_{\ell}=\left[\mathcal{H}_{2 \ell}^{2^{k}}\left(x_{2 \ell}\right)-\mathcal{H}_{2 \ell}^{2^{k}}\left(x_{\ell}\right)+O\left((2 e)^{2^{k}} \frac{\bar{x}^{\ell}}{\ell !}\right)\right] \mathbf{1}_{\ell},
$$

when $\ell \geq 64$. Now, let $A$ and $B$ be square matrices, then for every $k$ :

$$
\left\|\left|A^{2^{k}}-B^{2^{k}}\right| \mathbf{1}\right\| \leq\left(\|A\|+\left\|B^{\top}\right\|\right)^{2^{k}-1}\||A-B| \mathbf{1}\|,
$$

where $|C|$ denotes the matrix whose entries are the absolute values of those ones of $C$. This identity can be proved by induction on $k$ starting by the equality $A^{2}-B^{2}=$ $A(A-B)+(A-B) B$ implying the claim for $k=1$.

Using this bound for $A=\mathcal{H}_{2 \ell}^{2^{k}}\left(x_{2 \ell}\right)$ and $B=\mathcal{H}_{2 \ell}^{2^{k}}\left(x_{\ell}\right)$, we get:

$$
\left\|\left|\mathcal{H}_{2 \ell}^{2^{k}}\left(x_{2 \ell}\right)-\mathcal{H}_{2 \ell}^{2^{k}}\left(x_{\ell}\right)\right| \mathbf{1}_{\ell}\right\| \leq(2 e)^{2^{k}-1}\left\|\left|\mathcal{H}_{2 \ell}\left(x_{2 \ell}\right)-\mathcal{H}_{2 \ell}\left(x_{\ell}\right)\right| \mathbf{1}_{\ell}\right\|
$$

Moreover,

$$
\mathcal{H}_{2 \ell}\left(x_{2 \ell}\right)-\mathcal{H}_{2 \ell}\left(x_{\ell}\right)=O\left(\ell\left|x_{2 \ell}-x_{\ell}\right|\right) J_{\ell}
$$

because the derivative $\left(x^{u} G_{2 j-u}^{(4 \ell)}(x)\right)^{\prime}=u x^{u-1} G_{2 j-u}^{(4 \ell)}(x)+x^{u} G_{2 j-u-1}^{(4 \ell)}(x)$ is bounded by $e(\ell+1)$ for $u \leq \ell, j \leq 2 \ell$ and $x \in[0,1]$. The claim follows by (54-56) and Lemma 7.

To fully exploit the identity in Lemma 9 we need to understand the behavior of the powers of $\mathcal{F}_{\ell}\left(x_{\ell}\right)$ when they are applied to the vector $\mathbf{1}_{\ell}$. According to the Perron result, this sequence converges to an eigenvector of the 1-eigenspace. We need to know the rate of this convergence; for this purpose we need a bound for the second (in size) eigenvalue of $\mathcal{F}_{\ell}\left(x_{\ell}\right)$. There is a large body of literature addressing this problem (such as [4], [6] and [17]); nevertheless, these general results, when applied to the matrix $\mathcal{F}_{\ell}\left(x_{\ell}\right)$, produce bounds that are too weak for our purpose. Thus, the following lemma is an essential step in our argument.

Lemma 10. Let $\ell \geq 32$. Then, the eigenvalues of $\mathcal{F}_{\ell}\left(x_{\ell}\right)$ not equal to 1 are smaller than $1 / 2$ in absolute value. 
Proof. Let $\bar{q}_{\ell}(x):=\operatorname{det}\left(\mathbb{I}_{\ell}-x \mathcal{F}_{\ell}\left(x_{\ell}\right)\right)$. According to the definition of $x_{\ell}, x=1$ is a root of $\bar{q}_{\ell}(x)$ and the claim is equivalent to the fact that every other root of $\bar{q}_{\ell}$ is greater than 2 in absolute value. We prove this fact as a consequence of the Rouché's theorem applied to the inequality:

$$
\left|\bar{q}_{\ell}(x)-\bar{q}_{32}(x)\right|<\left|\bar{q}_{32}(x)\right| \quad|x|=2
$$

that we prove using an approach similar to that one for Lemma 3, and the circumstance that 1 is the unique root of $\bar{q}_{32}(x)$ having an absolute value lower than 2 . More details will be given later.

We recall the decomposition $\mathcal{F}_{\ell}(x)=\mathcal{A}_{\ell}^{\top}\left(x^{2}\right)+\mathcal{B}_{\ell}(x)$ that we already used for the proof of Lemma 7 . The matrices $\mathcal{A}_{\ell+1}^{\top}\left(x^{2}\right)$ and $\mathcal{A}_{\ell}^{\top}\left(x^{2}\right)$ are quite similar; therefore, we compute $\bar{q}_{\ell+1}(x)$ putting in evidence the part $\mathbb{I}_{\ell+1}-x \mathcal{A}_{\ell+1}^{\top}\left(x_{\ell+1}^{2}\right)$, which is similar to the analogous part appearing in $\bar{q}_{\ell}(x)$, and we write:

$$
\bar{q}_{\ell+1}(x)=\operatorname{det}\left(\mathbb{I}_{\ell+1}-x \mathcal{F}_{\ell}\left(x_{\ell+1}\right)\right)=\operatorname{det}\left(\mathbb{I}_{\ell+1}-x \mathcal{A}_{\ell+1}^{\top}\left(x_{\ell}^{2}\right)+\mathcal{C}_{\ell+1}(x)\right)
$$

where

$$
\mathcal{C}_{\ell+1}(x):=x \mathcal{A}_{\ell+1}^{\top}\left(x_{\ell}^{2}\right)-x \mathcal{A}_{\ell+1}^{\top}\left(x_{\ell+1}^{2}\right)-x \mathcal{B}_{\ell+1}\left(x_{\ell+1}\right) .
$$

For $\ell \geq 16$ we can assume $x_{\ell} \leq \bar{x}$, by Lemma 7 . Therefore, the $u, j$ entry in $\mathcal{C}_{\ell+1}$ is bounded by:

$$
|x|\left(\frac{\left|x_{\ell+1}^{2 j}-x_{\ell}^{2 j}\right|}{(2 j-u) !}+\frac{2 \bar{x}^{2 j+2 \ell+2}}{(2 j-u+2 \ell+2) !}\right)
$$

and since $2 j \bar{x}^{2 j-1}<4.63$ for every $j$, we deduce that each entry is bounded by:

$$
\begin{aligned}
& |x|\left(4.63\left|x_{\ell+1}-x_{\ell}\right|+\frac{2 \bar{x}^{4+2 \ell}}{(\ell+3) !}\right) \\
& \leq|x|\left(4.63 \cdot 1.7\left(\frac{\bar{x}^{2} e}{\sqrt{\ell}}\right)^{\ell}+4.63 \cdot 1.7\left(\frac{\bar{x}^{2} e}{\sqrt{\ell+1}}\right)^{\ell+1}+\frac{2 \bar{x}^{4+2 \ell}}{(\ell+3) !}\right) \\
& \leq 16|x|\left(\frac{\bar{x}^{2} e}{\sqrt{\ell}}\right)^{\ell},
\end{aligned}
$$

a quantity that for convenience we denote by $\lambda$. Hence,

$$
\bar{q}_{\ell+1}(x)=\operatorname{det}\left(\mathbb{I}_{\ell+1}-x \mathcal{A}_{\ell+1}^{\top}\left(x_{\ell}^{2}\right)+\bar{O}(\lambda) J_{\ell+1}\right) .
$$

Now we expand the determinant in powers of $\lambda$. The coefficient of $\lambda^{k}$ is bounded by the sum of the determinants of the principal minors of $\mathbb{I}_{\ell+1}-x \mathcal{A}_{\ell+1}^{\top}\left(x_{\ell}^{2}\right)$ having dimension $\ell+1-k$. Each minor can be estimated via the Hadamard bound as:

$\leq \prod_{j \in \text { minor }}\left(1+|x| \| j\right.$ th column in $\left.\mathcal{A}^{\top}\left(x_{\ell}^{2}\right) \|_{2}\right) \leq \prod_{j=1}^{\ell+1}\left(1+|x| \| j\right.$ th column in $\left.\mathcal{A}^{\top}\left(x_{\ell}^{2}\right) \|_{2}\right)$.

Since

$$
\| j \text { th column in } \mathcal{A}^{\top}\left(x_{\ell}^{2}\right) \|_{2} \leq x_{\ell}^{2 j}\left(\sum_{u=1}^{\ell+1} 1 /(2 j-u) !^{2}\right)^{1 / 2} \leq x_{\ell}^{2 j} \sqrt{e},
$$

each minor is bounded by:

$$
\prod_{j=1}^{\ell+1}\left(1+|x| x_{\ell}^{2 j} \sqrt{e}\right) \leq \prod_{j=1}^{\ell+1} \exp \left(|x| x_{\ell}^{2 j} \sqrt{e}\right) \leq \exp \left(\frac{|x| \bar{x}^{2} \sqrt{e}}{1-\bar{x}^{2}}\right)
$$


so that:

$$
\begin{aligned}
\bar{q}_{\ell+1}(x) & =\operatorname{det}\left(\mathbb{I}_{\ell+1}-x \mathcal{A}_{\ell+1}^{\top}\left(x_{\ell}^{2}\right)\right)+\bar{O}\left(\sum_{k=1}^{\ell+1} \lambda^{k}\left(\begin{array}{c}
\ell+1 \\
k
\end{array}\right) \exp \left(\frac{|x| \bar{x}^{2} \sqrt{e}}{1-\bar{x}^{2}}\right)\right) \\
& =\operatorname{det}\left(\mathbb{I}_{\ell+1}-x \mathcal{A}_{\ell+1}^{\top}\left(x_{\ell}^{2}\right)\right)+\bar{O}\left(\left((1+\lambda)^{\ell+1}-1\right) \exp \left(\frac{|x| \bar{x}^{2} \sqrt{e}}{1-\bar{x}^{2}}\right)\right) .
\end{aligned}
$$

Computing $\operatorname{det}\left(\mathbb{I}_{\ell+1}-x \mathcal{A}_{\ell+1}^{\top}\left(x_{\ell}^{2}\right)\right)$ via the Laplace formula with respect to the last column, we get $\operatorname{det}\left(\mathbb{I}_{\ell}-x \mathcal{A}_{\ell}^{\top}\left(x_{\ell}^{2}\right)\right.$ ) (which is obtained as cofactor of the $\ell+1, \ell+1$ entry), plus a quantity $R$ coming from the contribute of the other terms in the last column, and that therefore can be bounded as:

$$
|R| \leq \sum_{u=1}^{\ell+1} \frac{\bar{x}^{2 \ell+2}}{(2 \ell+2-u) !} \cdot \mid \text { cofactor of } u, \ell+1 \text { entry } \mid \text {. }
$$

The previous argument shows that the cofactor is bounded by $\exp \left(\frac{|x| \bar{x}^{2} \sqrt{e}}{1-\bar{x}^{2}}\right)$, thus:

$$
|R| \leq \frac{e \bar{x}^{2 \ell+2}}{(\ell+1) !} \exp \left(\frac{|x| \bar{x}^{2} \sqrt{e}}{1-\bar{x}^{2}}\right) \leq\left(\frac{\bar{x}^{2} e}{\ell}\right)^{\ell} \exp \left(\frac{|x| \bar{x}^{2} \sqrt{e}}{1-\bar{x}^{2}}\right)
$$

In this way we have proved that:

$$
\bar{q}_{\ell+1}(x)-\operatorname{det}\left(\mathbb{I}_{\ell}-x \mathcal{A}_{\ell}^{\top}\left(x_{\ell}^{2}\right)\right)=\bar{O}\left(\left((1+\lambda)^{\ell+1}-1+\left(\frac{\bar{x}^{2} e}{\ell}\right)^{\ell}\right) \exp \left(\frac{|x| \bar{x}^{2} \sqrt{e}}{1-\bar{x}^{2}}\right)\right) .
$$

An analogous argument shows that:

$$
\bar{q}_{\ell}(x)=\operatorname{det}\left(\mathbb{I}_{\ell}-x \mathcal{A}_{\ell}^{\top}\left(x_{\ell}^{2}\right)\right)+\bar{O}\left(\left(\left(1+\lambda^{\prime}\right)^{\ell}-1\right) \exp \left(\frac{|x| \bar{x}^{2} \sqrt{e}}{1-\bar{x}^{2}}\right)\right)
$$

with

By (58-59) we get:

$$
\lambda^{\prime}:=\frac{2 \bar{x}^{2}}{e(\ell+1)^{2}}\left(\frac{\bar{x}^{2} e}{\ell}\right)^{\ell}
$$

$$
\left|\bar{q}_{\ell+1}(x)-\bar{q}_{\ell}(x)\right| \leq\left(\left((1+\lambda)^{\ell+1}-1\right)+\left(\frac{\bar{x}^{2} e}{\ell}\right)^{\ell}+\left(\left(1+\lambda^{\prime}\right)^{\ell}-1\right)\right) \exp \left(\frac{|x| \bar{x}^{2} \sqrt{e}}{1-\bar{x}^{2}}\right) .
$$

Adding these inequalities we get:

$$
\left|\bar{q}_{\ell}(x)-\bar{q}_{32}(x)\right| \leq 0.02 \quad|x|=2,
$$

for every $\ell \geq 32$.

On the other hand, the polynomial $\bar{q}_{32}(x)$ can be computed explicitly (with PARIgp), and only the powers $\leq 3$ have significatively large coefficients. In fact,

$$
\bar{q}_{32}(x)=1-1.313 x+0.326 x^{2}-0.013 x^{3}+\bar{O}\left(7 \cdot 10^{-3}\right), \quad|x|=2 .
$$

It is now easy to verify that $\left|\bar{q}_{32}(x)\right| \geq 0.4$ along the circle $|x|=2$ so that (57) follows by (60). At last, a new application of the Rouché theorem proves that $\bar{q}_{32}(x)$ has a unique root below 2 , since the roots of $1-1.313 x+0.326 x^{2}-0.013 x^{3}$ are $1, \approx 3.79$ and $\approx 20.28$.

Now we have at our disposal the tools to understand the convergence of $\mathcal{F}^{2^{k}}\left(x_{\ell}\right) \mathbf{1}_{\ell}$. Numerical tests suggest that the eigenvalues of $\mathcal{F}_{\ell}\left(x_{\ell}\right)$ are real, positive, and simple, but we are unable to prove it in general. Lacking proof of these properties, our argument is a bit more complicated. 
Let $\boldsymbol{v}_{1}$ denote a 1-eigenvector of $\mathcal{F}_{\ell}\left(x_{\ell}\right)$, with real and positive entries. Let $\epsilon>0$ be an arbitrary constant, and let $\mathcal{R}_{\ell}=\mathcal{R}_{\ell}(\epsilon)$ be a square matrix such that:

i) $\mathcal{R}_{\ell} \boldsymbol{v}_{1}=0$.

ii) $\left\|\mathcal{R}_{\ell}\right\| \leq 1$.

iii) Let $\eta_{j, \epsilon}$ for $j=1, \ldots, \ell$ denote the eigenvalues of $\mathcal{F}_{\ell}\left(x_{\ell}\right)+\epsilon \mathcal{R}_{\ell}$, with $\eta_{1, \epsilon}=1$ for every $\epsilon$, then $\left|\eta_{j, \epsilon}-\eta_{j, 0}\right| \leq 10^{-2}$ for every $j$.

iv) The eigenvalues $\eta_{j, \epsilon}$ are simple.

Such a matrix $\mathcal{R}_{\ell}$ exists. In fact, the orthogonal complement of $\boldsymbol{v}_{1}$ is preserved by $\mathcal{F}_{\ell}\left(x_{\ell}\right)$; hence, we can identify the matrices satisfying $i$ with the matrices acting on $\boldsymbol{v}_{1}^{\perp} \rightarrow \boldsymbol{v}_{1}^{\perp}$. Under this identification and based on continuity the conditions $i i$ and iii are satisfied by any matrix in a suitable open neighborhood of the null matrix, and the matrices failing $i v$ belong to a closed and zero-measured subset.

For every $j \geq 2$, let $\boldsymbol{v}_{j, \epsilon}$ be an eigenvector of $\mathcal{F}_{\ell}\left(x_{\ell}\right)+\epsilon \mathcal{R}_{\ell}$ with eigenvalue $\eta_{j, \epsilon}$. Let

$$
\mathbf{1}_{\ell}=a_{1} \boldsymbol{v}_{1}+\sum_{k=2}^{\ell} a_{j} \boldsymbol{v}_{j, \epsilon}
$$

be the decomposition of $\mathbf{1}_{\ell}$ in the basis of the eigenvectors $\boldsymbol{v}_{j, \epsilon}$. Being simple, the orthogonality of the distinct eigenvectors gives the equality $a_{j}\left\|\boldsymbol{v}_{j, \epsilon}\right\|_{2}^{2}=\left\langle\mathbf{1}_{\ell}, \boldsymbol{v}_{j, \epsilon}\right\rangle$, for every $j$. Moreover, $\left\langle\mathbf{1}_{\ell}, \boldsymbol{v}_{1}\right\rangle=\left\|\boldsymbol{v}_{1}\right\|$ (because the entries of $\boldsymbol{v}_{1}$ are nonnegative), and $\left|\left\langle\mathbf{1}_{\ell}, \boldsymbol{v}_{j, \epsilon}\right\rangle\right| \leq\left\|\boldsymbol{v}_{j, \epsilon}\right\|$ in general, therefore:

$$
a_{1}=\frac{\left\|\boldsymbol{v}_{1}\right\|}{\left\|\boldsymbol{v}_{1}\right\|_{2}^{2}}, \quad\left|a_{j}\right| \leq \frac{\left\|\boldsymbol{v}_{j, \epsilon}\right\|}{\left\|\boldsymbol{v}_{j, \epsilon}\right\|_{2}^{2}} \quad \forall j .
$$

We have proved that the eigenvalues of $\mathcal{F}_{\ell}\left(x_{\ell}\right)$ are lower than $1 / 2$ in absolute value when $\ell \geq 32$, therefore for such $\ell$ and for every $k$ we have by $i-i v$ :

$$
\left(\mathcal{F}_{\ell}\left(x_{\ell}\right)+\epsilon \mathcal{R}_{\ell}\right)^{2^{k}} \mathbf{1}_{\ell}=\frac{\left\|\boldsymbol{v}_{1}\right\|}{\left\|\boldsymbol{v}_{1}\right\|_{2}^{2}} \boldsymbol{v}_{1}+O\left(\sum_{j=2}^{\ell} \frac{\left\|\boldsymbol{v}_{j, \epsilon}\right\|^{2}}{\left\|\boldsymbol{v}_{j, \epsilon}\right\|_{2}^{2}} 0.51^{2^{k}}\right) \mathbf{1}_{\ell} .
$$

The Cauchy-Schwarz inequality gives $\left\|\boldsymbol{v}_{j, \epsilon}\right\| \leq \sqrt{\ell}\left\|\boldsymbol{v}_{j, \epsilon}\right\|_{2}$, hence:

$$
\left(\mathcal{F}_{\ell}\left(x_{\ell}\right)+\epsilon \mathcal{R}_{\ell}\right)^{2^{k}} \mathbf{1}_{\ell}=\frac{\left\|\boldsymbol{v}_{1}\right\|}{\left\|\boldsymbol{v}_{1}\right\|_{2}^{2}} \boldsymbol{v}_{1}+O\left(\ell^{2} 0.51^{2^{k}}\right) \mathbf{1}_{\ell}
$$

RHS here is independent of $\epsilon$, therefore setting $\epsilon \rightarrow 0$ we conclude that:

$$
\mathcal{F}_{\ell}^{2^{k}}\left(x_{\ell}\right) \mathbf{1}_{\ell}=\frac{\left\|\boldsymbol{v}_{1}\right\|}{\left\|\boldsymbol{v}_{1}\right\|_{2}^{2}} \boldsymbol{v}_{1}+O\left(\ell^{2} 0.51^{2^{k}}\right) \mathbf{1}_{\ell}
$$

Let $\boldsymbol{\alpha}^{(\ell)}$ denote the eigenvector $\frac{\left\|\boldsymbol{v}_{1}\right\|}{\left\|\boldsymbol{v}_{1}\right\|_{2}^{2}} \boldsymbol{v}_{1}$. Then, the previous equality and Lemma 9 give:

$$
\boldsymbol{\alpha}^{(2 \ell)+}-\boldsymbol{\alpha}^{(\ell)}=O\left(\ell^{2} 0.51^{2^{k}}+\ell^{2}(2 e)^{2^{k}}\left(\frac{\bar{x}^{2} e}{\sqrt{\ell}}\right)^{\ell}\right) \mathbf{1}_{\ell}
$$

for every $\ell \geq 64$ and for every $k$. By choosing $k$ in such a way that $0.51^{2^{k}} \approx$ $(2 e)^{2^{k}}\left(\bar{x}^{2} e / \sqrt{\ell}\right)^{\ell}$ in $(61)$ we get:

$$
\boldsymbol{\alpha}^{(2 \ell)+}-\boldsymbol{\alpha}^{(\ell)}=O\left(\ell^{-0.14 \ell}\right) \mathbf{1}_{\ell},
$$

when $\ell$ is large enough. We complete $\boldsymbol{\alpha}^{(\ell)}$ to an infinite sequence by queueing infinitely many zeros. Suppose that $\ell$ diverges along the 2 powers; then (62) proves 
that there exists a new sequence $\boldsymbol{\alpha}^{(\infty)}$ of nonnegative numbers such that $\boldsymbol{\alpha}_{j}^{(\ell)} \rightarrow \boldsymbol{\alpha}_{j}^{(\infty)}$ for every fixed $j$. The following argument proves that the convergence is not only termwise, but also in the $\ell^{1}(\mathbb{N})$-norm: this claim needs an ad hoc proof, since (62) involves only the 'plus' part of $\boldsymbol{\alpha}^{(2 \ell)}$.

The relation $\mathcal{F}_{2 \ell}\left(x_{2 \ell}\right) \boldsymbol{\alpha}^{(2 \ell)}=\boldsymbol{\alpha}^{(2 \ell)}$ implies that:

$$
\mathcal{I}_{2 \ell}\left(x_{2 \ell}\right) \boldsymbol{\alpha}^{(2 \ell)+}+\mathcal{L}_{2 \ell}\left(x_{2 \ell}\right) \boldsymbol{\alpha}^{(2 \ell)-}=\boldsymbol{\alpha}^{(2 \ell)-} .
$$

The norm of $\mathcal{L}_{2 \ell}\left(x_{2 \ell}\right)$ is lower than $x_{2 \ell}^{2 \ell}$; in particular, it is lower than 1 so that $\mathbb{I}_{2 \ell}-\mathcal{L}_{2 \ell}\left(x_{2 \ell}\right)$ is invertible with inverse equal to $\sum_{k=0}^{+\infty} \mathcal{L}_{2 \ell}^{k}\left(x_{2 \ell}\right)$ and norm lower than $\left(1-x_{2 \ell}^{2 \ell}\right)^{-1}$. Hence $(63)$ can be solved for $\boldsymbol{\alpha}^{(2 \ell)-}$, giving:

$$
\boldsymbol{\alpha}^{(2 \ell)-}=\left(1-\mathcal{L}_{2 \ell}\left(x_{2 \ell}\right)\right)^{-1} \mathcal{I}_{2 \ell}\left(x_{2 \ell}\right) \boldsymbol{\alpha}^{(2 \ell)+}
$$

and

$$
\left\|\boldsymbol{\alpha}^{(2 \ell)-}\right\| \leq \frac{e}{1-x_{2 \ell}^{2 \ell}} x_{2 \ell}^{\ell}\left\|\boldsymbol{\alpha}^{(2 \ell)+}\right\| \leq 2 e x_{2 \ell}^{\ell}\left\|\boldsymbol{\alpha}^{(2 \ell)+}\right\| \quad \forall \ell \geq 64
$$

As a consequence, by (62):

$$
\begin{aligned}
& \left\|\boldsymbol{\alpha}^{(2 \ell)}\right\|=\left\|\boldsymbol{\alpha}^{(2 \ell)+}\right\|+\left\|\boldsymbol{\alpha}^{(2 \ell)-}\right\| \leq\left(1+2 e x_{2 \ell}^{\ell}\right)\left\|\boldsymbol{\alpha}^{(2 \ell)+}\right\| \\
& \leq\left(1+2 e \bar{x}^{\ell}\right)\left(\left\|\boldsymbol{\alpha}^{(\ell)}\right\|+b \ell^{-c \ell}\right)
\end{aligned}
$$

for a couple of positive constants $b$ and $c$, when $\ell \geq 64$. When written for the sequence of 2 powers this recursive bound implies that:

$$
\left\|\boldsymbol{\alpha}^{\left(2^{k+1}\right)}\right\| \leq\left(1+2 e \bar{x}^{2^{k}}\right)\left(\left\|\boldsymbol{\alpha}^{\left(2^{k}\right)}\right\|+b 2^{-c k 2^{k}}\right)
$$

giving

$$
\left\|\boldsymbol{\alpha}^{\left(2^{k}\right)}\right\| \leq\left\|\boldsymbol{\alpha}^{\left(2^{k}\right)}\right\| \prod_{s=4}^{k-1}\left(1+2 e \bar{x}^{2^{s}}\right)+b \sum_{s=4}^{k-1} 2^{-c s 2^{s}} \prod_{w=s+1}^{k-1}\left(1+2 e \bar{x}^{2^{w}}\right)
$$

for every $k \geq 5$. The convergence of the infinite product and of the series here above prove that the norms $\left\|\boldsymbol{\alpha}^{(\ell)}\right\|$ are uniformly bounded when $\ell$ runs along the 2 powers. From (65) we deduce that $\boldsymbol{\alpha}^{(2 \ell)-} \ll \bar{x}^{\ell}$ so that (by (62)):

$$
\left\|\boldsymbol{\alpha}^{(2 \ell)}-\boldsymbol{\alpha}^{(\ell)}\right\| \ll \ell\left(\ell^{-0.14 \ell}+\bar{x}^{\ell}\right) .
$$

This shows that the vectors $\left\{\boldsymbol{\alpha}^{(\ell)}\right\}_{\ell \text { power of } 2}$ form a Cauchy sequence in $\ell^{1}(\mathbb{N})$ and in particular that $\boldsymbol{\alpha}^{(\infty)}$ itself is summable.

Now we prove that the first entry in $\boldsymbol{\alpha}^{(\infty)}$ is strictly positive, later we will see that this implies that each entry in $\boldsymbol{\alpha}^{(\infty)}$ is strictly positive as well.

Lemma 11. $\boldsymbol{\alpha}_{1}^{(\infty)}>0$.

Proof. Let $\boldsymbol{\beta}^{(\ell)}$ be the positive eigenvector of $\mathcal{F}_{\ell}^{\top}\left(x_{\ell}\right)$, normalized in such a way that $\left\langle\boldsymbol{\beta}^{(\ell)}, \boldsymbol{\alpha}^{(\ell)}\right\rangle=1$. Then $\mathcal{F}_{\ell}^{2^{k}}\left(x_{\ell}\right)$ for $k \rightarrow \infty$ converges to $\boldsymbol{\alpha}^{(\ell)} \boldsymbol{\beta}^{(\ell) \top}$ (see [7], Th. 8.2.11); in particular:

$$
\left\|\boldsymbol{\alpha}^{(\ell)}\right\|=\left\|\mathcal{F}_{\ell}^{\infty}\left(x_{\ell}\right) \mathbf{1}_{\ell}\right\|=\left\|\boldsymbol{\alpha}^{(\ell)} \boldsymbol{\beta}^{(\ell) \top} \mathbf{1}_{\ell}\right\|=\sum_{j, j^{\prime}} \boldsymbol{\alpha}_{j}^{(\ell)} \boldsymbol{\beta}_{j^{\prime}}^{(\ell)} \geq\left\langle\boldsymbol{\beta}^{(\ell)}, \boldsymbol{\alpha}^{(\ell)}\right\rangle=1
$$


Moreover, the first line in $\mathcal{F}_{\ell}\left(x_{\ell}\right) \boldsymbol{\alpha}^{(\ell)}=\boldsymbol{\alpha}^{(\ell)}$ reads $\sum_{j=1}^{\ell} x_{\ell} G_{2 j-1}^{(2 \ell)}\left(x_{\ell}\right) \boldsymbol{\alpha}_{j}^{(\ell)}=\boldsymbol{\alpha}_{1}^{(\ell)}$. Using Lemma 7 and (66) it is easy to verify that this relation as $\ell$ diverges along powers of 2 becomes:

$$
\sum_{j=1}^{+\infty} \frac{\lambda_{\infty}^{-j}}{(2 j-1) !} \boldsymbol{\alpha}_{j}^{(\infty)}=\boldsymbol{\alpha}_{1}^{(\infty)}
$$

Suppose $\boldsymbol{\alpha}_{1}^{(\infty)}=0$. Then the previous equality implies that $\boldsymbol{\alpha}_{j}^{(\infty)}=0$ for every $j$, because we know that $\boldsymbol{\alpha}_{j}^{(\infty)} \geq 0$, which nevertheless contradicts (67).

Remark. With PARIgp we have computed $\boldsymbol{\alpha}_{1}^{(64)}$ and verified that it is $>0.95$. Thus, keeping track of the constants in (62) it is possible to prove that $\boldsymbol{\alpha}_{1}^{(\ell)} \geq 0.94$ for every 2-power $\ell$ greater than 64 .

From now on, we assume that $\ell$ is a 2 power, in order to take advantage of the convergence of $\boldsymbol{\alpha}^{(\ell)}$ to $\boldsymbol{\alpha}^{(\infty)}$ and of the uniform boundness of their norms. Under this hypothesis we prove a positive lower bound for the entries of $\boldsymbol{\alpha}^{(\ell)}$.

\section{Lemma 12.}

$$
\boldsymbol{\alpha}_{u}^{(\ell)} \gg x_{\ell}^{2 u+2\left\lceil\log _{2} u\right\rceil} \quad \forall \ell, \forall u \leq \ell .
$$

Proof. The $u$ th row in $\mathcal{F}_{\ell}\left(x_{\ell}\right) \boldsymbol{\alpha}^{(\ell)}=\boldsymbol{\alpha}^{(\ell)}$ reads:

$$
\boldsymbol{\alpha}_{u}^{(\ell)}=\sum_{j=1}^{\ell} x_{\ell}^{u} G_{2 j-u}^{(2 \ell)}\left(x_{\ell}\right) \boldsymbol{\alpha}_{j}^{(\ell)} .
$$

Thus, isolating the contribution of the term with $j=\lceil u / 2\rceil$ and recalling that $G_{0}^{(2 \ell)}\left(x_{\ell}\right)>1, G_{1}^{(2 \ell)}\left(x_{\ell}\right)>x_{\ell}$, we get:

$$
\boldsymbol{\alpha}_{u}^{(\ell)} \geq x_{\ell}^{u} G_{2\lceil u / 2\rceil-u}^{(2 \ell)}\left(x_{\ell}\right) \boldsymbol{\alpha}_{\lceil u / 2\rceil}^{(\ell)} \geq x_{\ell}^{2\lceil u / 2\rceil} \boldsymbol{\alpha}_{\lceil u / 2\rceil}^{(\ell)}, \quad \forall u \leq \ell .
$$

Iterating this inequality we obtain:

$$
\boldsymbol{\alpha}_{u}^{(\ell)} \geq x_{\ell}^{2 \sum_{k=1}^{\left\lceil\log _{2} u\right\rceil}\left\lceil u / 2^{k}\right\rceil} \boldsymbol{\alpha}_{1}^{(\ell)} \geq x_{\ell}^{2 u+2\left\lceil\log _{2} u\right\rceil} \boldsymbol{\alpha}_{1}^{(\ell)}, \quad \forall u \leq \ell,
$$

which is the claim since $\boldsymbol{\alpha}_{1}^{(\ell)}$ is bounded from below by a positive constant, by Lemma 11.

We use the uniform bound for the norms $\left\|\boldsymbol{\alpha}^{(\ell)}\right\|$ to prove an upper bound for the 'minus' components of $\boldsymbol{\alpha}^{(\ell)}$, stronger than the previous one in (65).

Lemma 13. There exist a positive constant $c$ and an integer $\ell_{0}$ such that:

$$
\boldsymbol{\alpha}_{u}^{(\ell)-} \ll \ell^{c} x_{\ell}^{\ell+2 u} \quad \forall \ell \geq \ell_{0}, \forall u \leq \ell / 2 .
$$

Proof. We split the proof in five steps.

Step 1. Let $\boldsymbol{\beta}:=\mathcal{I}_{\ell}\left(x_{\ell}\right) \boldsymbol{\alpha}^{(\ell)+}$. Then $\boldsymbol{\beta}_{u} \ll x_{\ell}^{\frac{\ell}{2}+u}$ for every $\ell$ and $u$.

In fact,

$$
\boldsymbol{\beta}_{u}=\sum_{j=1}^{\ell / 2} x_{\ell}^{\frac{\ell}{2}+u} G_{2 j-\frac{\ell}{2}-u}^{(2 \ell)}\left(x_{\ell}\right) \boldsymbol{\alpha}_{j}^{(\ell)+} .
$$

The uniform boundness of $\left\|\boldsymbol{\alpha}^{(\ell)}\right\|$ and the bound $\sum_{j=1}^{\ell / 2} G_{2 j-\frac{\ell}{2}-u}^{(2 \ell)}\left(x_{\ell}\right) \leq e^{x_{\ell}}$ give the claim. 
Step 2. Prove that $\boldsymbol{\alpha}_{u}^{(\ell)-} \ll x_{\ell}^{\ell / 2+u}$, for every $\ell$ and $u \leq \ell / 2$.

In fact, (64) gives:

$$
\boldsymbol{\alpha}^{(\ell)-}=\boldsymbol{\beta}+\left(1-\mathcal{L}_{\ell}\left(x_{\ell}\right)\right)^{-1} \mathcal{L}_{\ell}\left(x_{\ell}\right) \boldsymbol{\beta} .
$$

We know that $\left\|\mathcal{L}_{\ell}\left(x_{\ell}\right)\right\| \leq x_{\ell}^{\ell}$ and $\boldsymbol{\beta} \ll x_{\ell}^{\ell / 2}$, therefore $\left\|\left(1-\mathcal{L}_{\ell}\left(x_{\ell}\right)\right)^{-1} \mathcal{L}_{\ell}\left(x_{\ell}\right) \boldsymbol{\beta}\right\|$ $\ll x_{\ell}^{3 \ell / 2}$, thus:

$$
\boldsymbol{\alpha}_{u}^{(\ell)-}=\boldsymbol{\beta}_{u}+O\left(x_{\ell}^{3 \ell / 2}\right) \ll x_{\ell}^{\ell / 2+u}
$$

because $u \leq \ell / 2$.

Step 3. Improve the bound on $\boldsymbol{\beta}$ to $\boldsymbol{\beta}_{u} \ll x_{\ell}^{\frac{3}{4}(\ell+2 u)}$, for every $\ell$ and $u$. In fact,

$$
\begin{aligned}
\boldsymbol{\beta}_{u}= & \sum_{j=1}^{\ell / 2} x_{\ell}^{\ell / 2+u} G_{2 j-\ell / 2-u}^{(2 \ell)}\left(x_{\ell}\right) \boldsymbol{\alpha}_{j}^{(\ell)+} \\
= & \sum_{j=1}^{\ell / 2} x_{\ell}^{\ell / 2+u} \sum_{\substack{t=0, t: \\
2 j-\ell / 2-u+2 \ell t \geq 0}}^{+\infty} \frac{x_{\ell}^{2 j-\ell / 2-u+2 \ell t}}{(2 j-\ell / 2-u+2 \ell t) !} \boldsymbol{\alpha}_{j}^{(\ell)+} \\
= & \sum_{j=1}^{\ell / 2} x_{\ell}^{\ell / 2+u} \frac{x_{\ell}^{2 j-\ell / 2-u}}{(2 j-\ell / 2-u) !} \delta_{2 j \geq \ell / 2+u} \boldsymbol{\alpha}_{j}^{(\ell)+} \\
& \quad+\sum_{j=1}^{\ell / 2} x_{\ell}^{\ell / 2+u} \sum_{t=0}^{+\infty} \frac{x_{\ell}^{2 j-\ell / 2-u+2 \ell+2 \ell t}}{(2 j-\ell / 2-u+2 \ell+2 \ell t) !} \boldsymbol{\alpha}_{j}^{(\ell)+} .
\end{aligned}
$$

The uniform boundness of $\left\|\boldsymbol{\alpha}^{(\ell)}\right\|$ and the bound $2 j-\ell / 2-u+2 \ell \geq \ell+2$ (because $u \leq \ell / 2$ and $j \geq 1$ ) give:

$$
\boldsymbol{\beta}_{u} \ll \frac{x_{\ell}^{2 \ell}}{\ell !}+x_{\ell}^{\ell / 2+u} \sum_{j \geq \frac{\ell}{4}+\frac{u}{2}}^{\ell / 2} \frac{\boldsymbol{\alpha}_{j}^{(\ell)+}}{(2 j-\ell / 2-u) !} .
$$

We know that $\boldsymbol{\alpha}^{(\ell)+}=\boldsymbol{\alpha}^{(\ell / 2)}+O\left((\ell / 2)^{-1.4 \ell / 2}\right) \mathbf{1}_{\ell / 2}$ by $(62)$, and for $j \geq \frac{\ell}{4}+\frac{u}{2}$ we have $\boldsymbol{\alpha}_{j}^{(\ell / 2)}=\boldsymbol{\alpha}_{j-\ell / 4}^{(\ell / 2)-} \ll x_{\ell / 2}^{\ell / 4+(j-\ell / 4)}=x_{\ell / 2}^{j}$, by Step 2. Therefore:

$$
\begin{aligned}
\boldsymbol{\beta}_{u} & \ll \frac{x_{\ell}^{2 \ell}}{\ell !}+(\ell / 2)^{-1.4 \ell / 2}+x_{\ell}^{\ell / 2+u} \sum_{j \geq \frac{\ell}{4}+\frac{u}{2}}^{\ell / 2} \frac{x_{\ell / 2}^{j}}{(2 j-\ell / 2-u) !} \\
& \ll \frac{x_{\ell}^{2 \ell}}{\ell !}+(\ell / 2)^{-1.4 \ell / 2}+x_{\ell}^{\ell / 2+u} x_{\ell / 2}^{\ell / 4+u / 2} .
\end{aligned}
$$

By Lemma $7, x_{\ell}-x_{\ell / 2}=O\left(\frac{\bar{x}^{2} e}{\sqrt{\ell / 2}}\right)^{\ell / 2}$ (for $\ell \geq 32$ ), therefore the claim follows.

Step 4. Using the argument in Step 2 we deduce that $\boldsymbol{\alpha}_{u}^{(\ell)-} \ll x_{\ell}^{\frac{3}{4}(\ell+2 u)}$.

Step 5. Using the bound in Step 4 for $\boldsymbol{\alpha}_{u}^{(\ell)-}$ in Step 3 we get $\boldsymbol{\beta}_{u} \ll x_{\ell}^{\left(1-2^{-3}\right)(\ell+2 u)}$ and then the analogous upper bound for $\boldsymbol{\alpha}_{u}$; iterating this procedure $k$ times 
we get:

$$
\boldsymbol{\alpha}_{u}^{(\ell)-} \ll q^{k} x_{\ell}^{\left(1-2^{-k}\right)(\ell+2 u)}
$$

where $q$ is a suitable constant which is independent of $k$ and can be made independent of $\ell$ and $u$ if $\ell$ is large enough (actually, $q=6$ works). Setting $k$ in such a way that $2^{k} \asymp \ell$ we get the claim.

Lemma 14. There exist a positive constant $c^{\prime}$ such that:

$$
\ell^{c^{\prime}} x_{\ell}^{\ell} \boldsymbol{\alpha}^{(\ell)+} \gg \boldsymbol{\alpha}^{(\ell)-} \text {. }
$$

Proof. By Lemmas 12 and 13 it is sufficient to prove that there exists $c^{\prime}>0$ such that:

$$
\ell^{c^{\prime}} x_{\ell}^{\ell} x_{\ell}^{2 u+2\left\lceil\log _{2} u\right\rceil} \gg \ell^{c} x_{\ell}^{\ell+2 u} \quad \forall u \leq \ell / 2 .
$$

Any $c^{\prime}$ which is greater than $c-2 \log _{2} x_{\ell}$ for every large $\ell\left(\right.$ e.g., $\left.c^{\prime}=c+1\right)$ works.

Finally we can prove our main result.

Lemma 15. There exists $\ell_{0}$ and a positive constant $\gamma$ such that:

$$
\overline{\mathcal{F}}_{\ell}\left(x_{\ell}^{\prime}\right) \boldsymbol{\alpha}^{(2 \ell)+} \leq \boldsymbol{\alpha}^{(2 \ell)+} \quad \forall \ell \geq \ell_{0}
$$

for $x_{\ell}^{\prime}:=x_{2 \ell}\left(1-\gamma \frac{\log _{2} \ell}{\ell}\right)$.

Proof. We look for an $x$ such that $\overline{\mathcal{F}}_{\ell}(x) \boldsymbol{\alpha}^{(2 \ell)+} \leq \boldsymbol{\alpha}^{(2 \ell)+}$, with $x-x_{2 \ell}=o(1)$. We know that $\overline{\mathcal{F}}_{\ell}=\mathcal{I}_{2 \ell}+\mathcal{L}_{2 \ell}$, therefore the inequality can be written as:

$$
\mathcal{I}_{2 \ell}(x) \boldsymbol{\alpha}^{(2 \ell)+}+\mathcal{L}_{2 \ell}(x) \boldsymbol{\alpha}^{(2 \ell)+} \leq \boldsymbol{\alpha}^{(2 \ell)+} .
$$

For every $x \leq x_{2 \ell}$ we have $\mathcal{I}_{2 \ell}(x) \boldsymbol{\alpha}^{(2 \ell)+} \leq \boldsymbol{\alpha}^{(2 \ell)-}$ by $(63)$, hence (68) is implied by:

$$
\boldsymbol{\alpha}^{(2 \ell)-} \leq \frac{1}{2} \boldsymbol{\alpha}^{(2 \ell)+}, \quad \mathcal{L}_{2 \ell}(x) \boldsymbol{\alpha}^{(2 \ell)+} \leq \frac{1}{2} \boldsymbol{\alpha}^{(2 \ell)+} .
$$

The first inequality does not involve $x$ and holds for $\ell$ large enough, by Lemma 14 . For the second one, we see that:

$$
\left[\mathcal{L}_{2 \ell}(x) \boldsymbol{\alpha}^{(2 \ell)+}\right]_{u}=\sum_{j=1}^{\ell} x^{\ell+u} G_{2(j+\ell)-\ell-u}^{(4 \ell)}(x) \boldsymbol{\alpha}_{j}^{(2 \ell)+} \ll x^{\ell+u},
$$

because the components $\boldsymbol{\alpha}_{j}^{(2 \ell)+}$ are bounded uniformly on $\ell$ and $j$. Recalling Lemma 12 , hence, it is sufficient to find $x \leq x_{2 \ell}$ such that:

$$
c x^{\ell+u} \leq x_{2 \ell}^{2 u+2\left\lceil\log _{2} u\right\rceil}
$$

holds for all $u \leq \ell$, where $c$ is a constant independent of $\ell$ and $u$. We meet this condition by choosing:

$$
x:=x_{2 \ell}\left(1-\gamma \frac{\log _{2} \ell}{\ell}\right),
$$

for a suitable positive constant $\gamma$ independent of $\ell$ and $u$. In fact, for such an $x$ the inequality becomes:

$$
x_{2 \ell}^{\ell-u-2\left\lceil\log _{2} u\right\rceil}\left(1-\gamma \frac{\log _{2} \ell}{\ell}\right)^{\ell+u} \leq 1 / c .
$$

The greatest value of LHS is attained for $u=\ell$ in the first factor and $u=0$ in the second one, therefore it is sufficient to have:

$$
x_{2 \ell}^{-2\left\lceil\log _{2} \ell\right\rceil}\left(1-\gamma \frac{\log _{2} \ell}{\ell}\right)^{\ell} \leq 1 / c .
$$


This inequality holds whenever $\ell$ is large enough if we set $\gamma>-2 \log x_{2 \ell}$ for every $\ell$ (and this is possible uniformly in $\ell$, by Lemma 7 ) because in this case LHS tends to 0 as $\ell$ diverges.

We can now conclude the proof of the theorem. By (62) the inequality in Lemma 15 can be written as:

$$
\overline{\mathcal{F}}_{\ell}\left(x_{\ell}^{\prime}\right) \boldsymbol{\alpha}^{(\ell)}+O\left(\ell^{-0.14 \ell}\right) \overline{\mathcal{F}}_{\ell}\left(x_{\ell}^{\prime}\right) \mathbf{1}_{\ell} \leq \boldsymbol{\alpha}^{(\ell)}+O\left(\ell^{-0.14 \ell}\right) \mathbf{1}_{\ell} \quad \forall \ell \geq \ell_{0} .
$$

Since $\overline{\mathcal{F}}_{\ell}\left(x_{\ell}^{\prime}\right) \mathbf{1}_{\ell} \leq\left\|\overline{\mathcal{F}}_{\ell}\left(x_{\ell}^{\prime}\right)^{\top}\right\| \mathbf{1}_{\ell} \ll \mathbf{1}_{\ell}$ independently of $\ell$, this means that:

$$
\overline{\mathcal{F}}_{\ell}\left(x_{\ell}^{\prime}\right) \boldsymbol{\alpha}^{(\ell)}+O\left(\ell^{-0.14 \ell}\right) \mathbf{1}_{\ell} \leq \boldsymbol{\alpha}^{(\ell)} \quad \forall \ell \geq \ell_{0} .
$$

Now we show that we can find $x_{\ell}^{\prime \prime} \leq x_{\ell}^{\prime}$ with $x_{\ell}^{\prime \prime}-x_{\ell}^{\prime}=o(1)$ and such that $\overline{\mathcal{F}}_{\ell}\left(x_{\ell}^{\prime \prime}\right) \boldsymbol{\alpha}^{(\ell)}$ is lower than LHS in (69). In fact, let $h \in(0,1 / 10)$ that we will fix later. Then

$$
\overline{\mathcal{F}}_{\ell}\left(x_{\ell}^{\prime}-h\right)=\overline{\mathcal{F}}_{\ell}\left(x_{\ell}^{\prime}\right)-h \mathcal{D}
$$

where $\mathcal{D}$ is a matrix whose $u, j$ entry is the derivative of $x^{u} G_{2 j-u}^{(2 \ell)}(x)$, and hence $u x^{u-1} G_{2 j-u}^{(2 \ell)}(x)+x^{u} G_{2 j-u-1}^{(2 \ell)}(x)$, computed at a point in $\left[x_{\ell}^{\prime}-h, x_{\ell}^{\prime}\right]$, and hence in $(1 / 2,1)$. Suppose $u$ even. Then the term with $j=u / 2$ is greater than $u x^{u-1} G_{0}^{(2 \ell)}(x)$ $\geq u x^{u-1} G_{0}^{(2 \ell)}(1 / 2) \geq 1 / 2^{u}$. If $u$ is odd the same happens to the term with $j=$ $(u+1) / 2$, therefore in every row of $\mathcal{D}$ there is an entry which is greater than $1 / 2^{u}$. This implies that:

$$
\overline{\mathcal{F}}_{\ell}\left(x_{\ell}^{\prime}-h\right) \boldsymbol{\alpha}^{(\ell)} \leq \overline{\mathcal{F}}_{\ell}\left(x_{\ell}^{\prime}\right) \boldsymbol{\alpha}^{(\ell)}-h \min _{u}\left\{\boldsymbol{\alpha}_{u}^{(\ell)}\right\}\left(\begin{array}{c}
1 / 2 \\
1 / 2^{2} \\
\ldots \\
1 / 2^{\ell}
\end{array}\right) .
$$

Therefore $x_{\ell}^{\prime \prime}:=x_{\ell}^{\prime}-h$ satisfies $\overline{\mathcal{F}}_{\ell}\left(x_{\ell}^{\prime \prime}\right) \boldsymbol{\alpha}^{(\ell)} \leq \boldsymbol{\alpha}^{(\ell)}$ whenever:

$$
h \min _{u}\left\{\boldsymbol{\alpha}_{u}^{(\ell)}\right\}\left(\begin{array}{c}
1 / 2 \\
1 / 2^{2} \\
\cdots \\
1 / 2^{\ell}
\end{array}\right) \geq c \ell^{-0.14 \ell} \mathbf{1}_{\ell}
$$

where $c$ is the fixed constant whose value is implicit in (69). Recalling Lemma 12, it is sufficient to set $h$ such that:

$$
h x_{\ell}^{2 \ell+2\left\lceil\log _{2} \ell\right\rceil}\left(\begin{array}{c}
1 / 2 \\
1 / 2^{2} \\
\cdots \\
1 / 2^{\ell}
\end{array}\right) \geq c^{\prime} \ell^{-0.14 \ell} \mathbf{1}_{\ell}
$$

and if we choose $h=1 / \ell$ this inequality is true for $\ell$ large enough.

Let $\check{x}_{\ell}:=\min \left\{x_{\ell}^{\prime \prime}, x_{\ell}\right\}$, then we have both

$$
\overline{\mathcal{F}}_{\ell}\left(\check{x}_{\ell}\right) \boldsymbol{\alpha}^{(\ell)} \leq \overline{\mathcal{F}}_{\ell}\left(x_{\ell}^{\prime \prime}\right) \boldsymbol{\alpha}^{(\ell)} \leq \boldsymbol{\alpha}^{(\ell)} \quad \text { and } \quad \mathcal{F}_{\ell}\left(\check{x}_{\ell}\right) \boldsymbol{\alpha}^{(\ell)} \leq \mathcal{F}_{\ell}\left(x_{\ell}\right) \boldsymbol{\alpha}^{(\ell)}=\boldsymbol{\alpha}^{(\ell)},
$$

for all $\ell \geq \ell_{0}$. Therefore any constant below $\check{x}_{\ell}$ satisfies (51), giving the upper bound $\Lambda_{\infty} \leq \check{x}_{\ell}^{-2}$ for every 2-power $\ell$, large enough. Since $\check{x}_{\ell} \rightarrow 1 / \sqrt{\lambda_{\infty}}$, we get that $\Lambda_{\infty} \leq \lambda_{\infty}$, which concludes the proof of the theorem.

The sequence $\mathcal{F}_{\ell}\left(x_{\ell}\right)$ can be made to a sequence of operators $\ell^{\infty}(\mathbb{N}) \rightarrow \ell^{1}(\mathbb{N})$, uniformly converging to the compact operator $\mathcal{F}: \ell^{\infty}(\mathbb{N}) \rightarrow \ell^{1}(\mathbb{N})$, whose $u, j$ entry is $\lambda_{\infty}^{-j} /(2 j-u)$ !. Restricting $\mathcal{F}$ to $\ell^{2}(\mathbb{N}) \rightarrow \ell^{2}(\mathbb{N})$, we get a new operator having 
$\boldsymbol{\alpha}^{(\infty)}$ as eigenvector, with an eigenvalue equal to 1 . This fact addresses the question of whether it is possible to use $\mathcal{F}$ and its eigenvector $\boldsymbol{\alpha}^{(\infty)}$ to prove that $\Lambda_{\infty} \leq \lambda_{\infty}$, perhaps as a consequence of the formula (29) for $d_{i, u}$. For example, we can try to prove a bound of the form $d_{i, u} \ll_{\eta} \boldsymbol{\alpha}_{u}^{(\infty)}\left(\lambda_{\infty}+\eta\right)^{i}$ for every $i$ and $u$, for every $\eta>0$. This bound is the natural extension of (47) and produces an immediate proof of the theorem, according to (31). Nevertheless, it would imply that:

$$
\left(\lambda_{\infty}+\eta\right)^{-i} \ll_{\eta} \boldsymbol{\alpha}_{2 i}^{(\infty)}
$$

because $d_{i, 2 i} \geq 1$, according to (29), which is impossible for $\eta<\lambda_{\infty}^{2}-\lambda_{\infty}$ since from Lemmas 12 and 13 it follows that:

$$
\lambda_{\infty}^{-u-\left\lceil\log _{2} u\right\rceil} \ll \boldsymbol{\alpha}_{u}^{(\infty)} \ll u^{c} \lambda_{\infty}^{-u} \quad \forall u
$$

for a suitable constant $c$.

These difficulties show, in some sense, why our proof has followed from a judicious use of the interpolating vectors $\boldsymbol{\alpha}^{(\ell)}$ and of some very tricky properties of them, and is not just based on the existence of $\mathcal{F}$ and $\boldsymbol{\alpha}^{(\infty)}$.

Acknowledgement. The author is indebted with the anonymous referee for a very interesting remark which has simplified the argument proving (11) and for having pointed to his attention the interesting results on the constants $b_{m, d}(\ell)$.

The author was funded by an NSF Grant DMS 0635607 during the period he has spent at the Institute for the Advanced Study in Princeton, where this work had been mainly conceived. He warmly thanks the Institute for its hospitality.

\section{REFERENCES}

[1] R. Bhatia, Matrix analysis, Graduate Texts in Mathematics, vol. 169, Springer-Verlag, New York, 1997.

[2] N. G. de Bruijn, On Mahler's partition problem, Nederl. Akad. Wetensch., Proc. 51 (1948), 659-669 = Indagationes Math. 10, 210-220 (1948).

[3] P. Erdős, On an elementary proof of some asymptotic formulas in the theory of partitions, Ann. of Math. (2) 43 (1942), 437-450.

[4] S. Friedland and L. Gurvits, An upper bound for the real part of nonmaximal eigenvalues of nonnegative irreducible matrices, SIAM J. Matrix Anal. Appl. 15 (1994), no. 3, 1015-1017.

[5] A. Giorgilli and G. Molteni, Representation of a 2-power as sum of $k$ 2-powers: a recursive formula, preprint, 2009.

[6] O. Gross and U. G. Rothblum, Approximations of the spectral radius corresponding eigenvector, and second largest modulus of an eigenvalue for square, nonnegative, irreducible matrices, SIAM J. Matrix Anal. Appl. 14 (1993), no. 1, 15-32.

[7] R. A. Horn and C. R. Johnson, Matrix analysis, Cambridge University Press, Cambridge, 1990.

[8] A. E. Ingham, A Tauberian theorem for partitions, Ann. of Math. (2) 42 (1941), 1075-1090.

[9] D. E. Knuth, An almost linear recurrence, Fibonacci Quart. 4 (1966), 117-128.

[10] S. Lehr, J. Shallit, and J. Tromp, On the vector space of the automatic reals, Theoret. Comput. Sci. 163 (1996), no. 1-2, 193-210.

[11] K. Mahler, On a special functional equation, J. London Math. Soc. 15 (1940), 115-123.

[12] G. Molteni, Cancellation in a short exponential sum, J. Number Theory 130 (2010), no. 9, 2011-2027.

[13] The PARI Group, Bordeaux, Pari/gp, version 2.2.10, 2004, available from http://pari. math.u-bordeaux.fr/.

[14] V. Yu. Protasov, Asymptotics of the partition function, Mat. Sb. 191 (2000), no. 3, 65-98.

[15] - On the problem of the asymptotics of the partition function, Mat. Zametki 76 (2004), no. $1,151-156$.

[16] B. Reznick, Some binary partition functions, Analytic number theory (Allerton Park, IL, 1989), Progr. Math., vol. 85, Birkhäuser Boston, Boston, MA, 1990, pp. 451-477. 
[17] U. G. Rothblum and C. P. Tan, Upper bounds on the maximum modulus of subdominant eigenvalues of nonnegative matrices, Linear Algebra Appl. 66 (1985), 45-86.

[18] W. Rudin, Real and complex analysis, third ed., McGraw-Hill Book Co., New York, 1987.

[19] R. S. Varga, Matrix iterative analysis, expanded ed., Springer Series in Computational Mathematics, vol. 27, Springer-Verlag, Berlin, 2000.

Dipartimento di Matematica, Università di Milano, via Saldini 50, I-20133 Milano, ITALY

E-mail address: giuseppe.molteni1@unimi.it 